\title{
GASP XXIX - unwinding the arms of spiral galaxies via ram-pressure stripping
}

\author{
Callum Bellhouse ${ }^{\circledR}, 1,2 \star$ Sean L. McGee, ${ }^{2}$ Rory Smith, ${ }^{3}$ Bianca M. Poggianti, ${ }^{1}$ Yara L. Jaffé ${ }^{\circledR},{ }^{4}$ \\ Katarina Kraljic ${ }^{\circledR}, 5$ Andrea Franchetto, ${ }^{1,6}$ Jacopo Fritz ${ }^{(0)},{ }^{7}$ Benedetta Vulcani ${ }^{\circledR},{ }_{1}$ Stephanie Tonnesen, ${ }^{8}$ \\ Elke Roediger, ${ }^{9}$ Alessia Moretti ${ }^{\circledR},{ }^{1}$ Marco Gullieuszik ${ }^{1}$ and Jihye Shin ${ }^{3}$ \\ ${ }^{1}$ INAF - Astronomical Observatory of Padova, vicolo dell'Osservatorio 5, I-35122 Padova, Italy \\ ${ }^{2}$ School of Physics and Astronomy, University of Birmingham, Edgbaston, Birmingham B15 2TT, UK \\ ${ }^{3}$ Korea Astronomy and Space Science Institute, 776, Daedeokdae-ro, Yuseong-gu, Daejeon 34055, Korea \\ ${ }^{4}$ Instituto de Física y Astronomía, Universidad de Valparaíso, Avda. Gran Bretaña 1111, Valparaíso 2360102, Chile \\ ${ }^{5}$ Royal Observatory Edinburgh, Blackford Hill, Edinburgh EH9 3HJ, UK \\ ${ }^{6}$ Dipartimento di Fisica and Astronomia 'Galileo Galilei', Università di Padova, vicolo dell' Osservatorio 3 , I-35122 Padova, Italy \\ ${ }^{7}$ Instituto de Radioastronomía y Astrofísica, UNAM, Campus Morelia, A.P. 3-72, C.P. 58089, Mexico \\ ${ }^{8}$ Center for Computational Astrophysics, Flatiron Institute, 162 5th Avenue, New York, NY 10010, USA \\ ${ }^{9}$ E.A. Milne Centre for Astrophysics, Department of Physics and Mathematics, University of Hull, Hull HU6 7RX, UK
}

Accepted 2020 October 16. Received 2020 September 4; in original form 2020 April 23

\begin{abstract}
We present the first study of the effect of ram pressure 'unwinding' the spiral arms of cluster galaxies. We study 11 ram-pressure stripped galaxies from GASP (GAs Stripping Phenomena in galaxies) in which, in addition to more commonly observed 'jellyfish' features, dislodged material also appears to retain the original structure of the spiral arms. Gravitational influence from neighbours is ruled out and we compare the sample with a control group of undisturbed spiral galaxies and simulated stripped galaxies. We first confirm the unwinding nature, finding that the spiral arm pitch angle increases radially in 10 stripped galaxies and also simulated face-on and edge-on stripped galaxies. We find only younger stars in the unwound component, while older stars in the disc remain undisturbed. We compare the morphology and kinematics with simulated ram-pressure stripping galaxies, taking into account the estimated inclination with respect to the intracluster medium (ICM) and find that in edge-on stripping, unwinding can occur due to differential ram pressure caused by the disc rotation, causing stripped material to slow and 'pile up'. In face-on cases, gas removed from the outer edges falls to higher orbits, appearing to 'unwind'. The pattern is fairly short-lived $(<0.5 \mathrm{Gyr})$ in the stripping process, occurring during first infall and eventually washed out by the ICM wind into the tail of the jellyfish galaxy. By comparing simulations with the observed sample, we find that a combination of face-on and edge-on 'unwinding' effects is likely to be occurring in our galaxies as they experience stripping with different inclinations with respect to the ICM.
\end{abstract}

Key words: galaxies: interactions-galaxies: kinematics and dynamics-galaxies: evolution-galaxies: clusters: generalgalaxies: ISM - galaxies: clusters: intracluster medium.

\section{INTRODUCTION}

The development and evolution of galaxies is heavily influenced by gas inflows and outflows, since gas is the fuel of star formation in galaxies. Understanding how gas behaves both within the disc and in the surroundings of a galaxy is therefore vital in building a complete picture of its evolution. Galaxies can experience changes in their gas content in isolation, due to internal effects such as winds from, e.g. Wolf-Rayet and OB-type stars, supernova feedback, and feedback from active galactic nucleus (AGN) activity (Veilleux, Cecil \& BlandHawthorn 2005; Ho et al. 2014). External environmental forces can also influence and, in some cases, dramatically alter the gas content within a galaxy (Boselli \& Gavazzi 2006). Within galaxy clusters, the largest gravitationally bound objects in the Universe, there are many environmental processes that can affect individual galaxies owing to the increased frequency of interactions with other galaxies, and also to interaction with the dense intracluster medium (ICM). As galaxies move through a cluster, they may experience gravitational and hydrodynamical effects. Gravitational effects, such as tidal interactions with the cluster (Byrd \& Valtonen 1990; Valluri 1993) and gravitational interactions between galaxies (Spitzer \& Baade 1951; Toomre 1977; Tinsley \& Larson 1979; Merritt 1983; Mihos, Bothun \& Richstone 1993; Springel 2000), affect both the stellar and gas components of a galaxy. Hydrodynamical effects resulting from the cluster ICM affect only the gas content of the galaxy, as the stars are too massive and compact to be susceptible to hydrodynamical influence.

One particular hydrodynamical effect that plays a key role in transforming and quenching galaxies in clusters is ram-pressure 
stripping (RPS; Gunn \& Gott 1972). In the process of RPS, the ICM interacts directly with the gas component of an infalling galaxy. The process can ultimately lead to complete removal of this gas as well as many associated observable effects including compression of gas along the leading edge of the disc (Rasmussen, Ponman \& Mulchaey 2006; Poggianti et al. 2019b), a boost in star formation during the peak stripping phase (Vulcani et al. 2018) followed by quenching in the final stages of stripping (Vulcani et al. 2020), the formation of tails of gas in the wake of the galaxy (Fumagalli et al. 2014; Cramer et al. 2019), and the condensation of star-forming knots in the tails (Kenney et al. 2014; Poggianti et al. 2019a).

The extended tails of jellyfish galaxies are easily seen in studies of the ionized gas, and can be narrow and collimated along the reverse of the direction of motion (Kenney \& Koopmann 1999; Fumagalli et al. 2014; Boselli et al. 2018; Fossati et al. 2018).

The GASP (GAs Stripping Phenomena in galaxies) survey (Poggianti et al. 2017b) utilizes the powerful Multi-Unit Spectroscopic Explorer (MUSE: Bacon et al. 2010) at the Very Large Telescope in Chile to study in detail a sample of 94 ram-pressure stripped galaxies drawn from the Poggianti et al. (2016) sample. These galaxies were selected by visual inspection based on the presence of RPS signatures, such as displaced tails and asymmetric morphologies, from the WIde-field Nearby Galaxy-cluster Survey (WINGS; Fasano et al. 2006; Moretti et al. 2014), its extension OmegaWINGS (Gullieuszik et al. 2015; Moretti et al. 2017), and the Padova-Millennium Galaxy and Group Catalogue (Calvi, Poggianti \& Vulcani 2011). The aim of the GASP survey is to explore and characterize the nature of the stripping process and its effect on a multitude of galaxies in different environments. This large sample has facilitated the opportunity to observe many new aspects and effects of RPS, including the possibility that RPS can funnel the gas into the central region of the galaxy, triggering and fuelling the AGN (Poggianti et al. 2017a), and the widespread evidence of in-situ star formation in the tails (Poggianti et al. 2019a). One of the most heavily studied jellyfish galaxies of the GASP survey to date has been JO201, analysed both individually in Bellhouse et al. (2017, 2019), George et al. (2018, 2019), and Ramatsoku et al. (2020) and as part of wider studies (Poggianti et al. 2017a; Moretti et al. 2018; Vulcani et al. 2018; Radovich et al. 2019). JO201 is a massive galaxy falling into the massive cluster Abell 85 at supersonic speeds close to the line of sight towards the observer, exhibiting signs of extreme near-face-on RPS. The spatial distribution of the stripped knots hinted for the first time at the possibility that the spiral arms could be 'unwound' or broadened as an effect of the face-on gas stripping by the ICM (see Bellhouse et al. 2017 and fig. 2 of George et al. 2018). In addition to the more commonly observed 'jellyfish' features such as compression of the disc and one-sided tails, the trailing knots and the ionized gas appear to be distributed along the curves of the spiral arms.

Since such observed structures in the tails resemble the tidal tails associated with gravitational interactions, it is paramount to understand the implications of such patterns being observed in galaxies undergoing RPS. In particular, is there a mechanism by which ram pressure alone can lead to the 'unwinding' feature, without the influence of gravitational interactions? Furthermore, since in many previous studies of jellyfish galaxies, such an effect has not been noted, is there a specific set of conditions under which this 'unwinding' takes place? In comparison to tidal interactions, the unwinding effect in ram-pressure stripped galaxies appears to be accompanied by other signatures of the interaction, such as tail features, compression of the disc, and the formation of trailing knots of ionized gas. While unwinding alone is not an indicator of RPS, we aim to understand what the unwinding morphologies can tell us about a galaxy undergoing the process. Furthermore, understanding the ability of RPS to induce such a pattern is important when considering sample selection from broad-band data. Since curved tails are typically associated with tidal interactions, potential ram-pressure stripped galaxies may be unduly excluded by visual inspection or automated feature detection, and in such cases further analysis may be required to constrain the true nature of the interaction. Since many studies of ram-pressure stripped galaxies, including the GASP survey, are based on visual identification of stripping features, it is crucial to ensure that this selection does not exclude a population of ram-pressure stripped galaxies that are being unwound, but show minimal to no signs of 'classic' RPS signatures.

In the literature, a limited number of examples of 'unwinding' effects in ram-pressure stripped galaxies can be found.

In Schulz \& Struck (2001), hydrodynamical simulations of faceon and inclined stripping galaxies reveal the formation of flocculent spiral arms in the non-stripped disc gas as the remnant disc is compressed. Furthermore, the simulations show stretching and shearing of outer spiral arms prior to their removal from the galaxy.

In Roediger et al. (2014), a simulated galaxy undergoing RPS edge-on to the ICM shows filamentary tails extending away from the disc as the spiral arm material is stripped. The galaxy was subjected to a piston-shock followed by a constant wind speed of $2000 \mathrm{kms}^{-1}$, resulting in the galaxy being stripped substantially, but not completely. The galaxy disc was truncated on the leading edge by the stripping and elongated on the trailing side. The authors point out that material tailing behind the galaxy is not a 'tail' in the conventional sense, but remains bound to the galaxy and rotates with the original disc. The unwinding effect is visible $185 \mathrm{Myr}$ after initial stripping and very prominent $263 \mathrm{Myr}$ after initial stripping in Fig. 2 of the paper.

Steinhauser, Schindler \& Springel (2016) carried out simulations of galaxies undergoing RPS under different conditions, with several cluster masses and orbital trajectories. Two of the galaxies in their sample appear to show the unwinding effect (see galaxies S1 and S4, fig. 12 therein), which are simulated to mimic galaxies undergoing extreme plunging orbits in their host clusters.

In the observational study of Wolter et al. (2015), the galaxy NGC 2276 was analysed and the source of morphological disturbance was investigated. At optical wavelengths, the galaxy exhibits a similar pattern to our sample, with loose spiral arms extended to one side of the galaxy. The authors concluded from estimation of the gravitational and hydrodynamical interaction strengths and from comparison with simulations that the galaxy will be most strongly affected by RPS. In the study of Tomičić et al. (2018), evidence of tidal interaction on NGC 2276 was observed in the large-scale gradient in the molecular gas depletion time across the disc of the galaxy. Such variations in the star formation efficiency of the molecular gas resulting from both tidal and ram-pressure interactions may also play a role in shaping the morphology of NGC 2276.

In this paper, we further investigate this effect in JO201 and in the wider sample of GASP galaxies, in the quest for more evidence and a greater understanding of spiral arm unwinding during RPS events.

This paper is structured as follows. Section 2 covers the preparation of the data used in this study, the selection process used to produce the sample, and the resulting galaxies used. In Section 3, we show the locations of the sample in projected position velocity phase space, along with their tail orientations and morphologies, using these to infer the orbits and inclinations of stripping for the galaxies in the sample. Section 4 shows the analysis of the MUSE data to measure the spiral arm structures and pitch angles, and to trace the distribution of different age stellar populations in the spiral arms. In Section 5, 
we show the analysis of simulated galaxies undergoing RPS with different orientations with respect to the ICM wind, comparing with observed galaxies, analysing the effect of unwinding over time throughout infall and studying the effect of stripping on the gas kinematics to understand how the unwinding patterns can arise. Finally, in Section 6 we summarize the results and draw conclusions.

Throughout this paper, as in other GASP studies, we adopt a Chabrier initial mass function (Chabrier 2003), and a concordance Lambda cold dark matter cosmology of $\Omega_{\mathrm{M}}=0.3, \Omega_{\Lambda}=0.7$, and $H_{0}=70 \mathrm{~km} \mathrm{~s}^{-1} \mathrm{Mpc}^{-1}$.

\section{THE SAMPLE AND DATA}

In order to investigate and understand the unwinding effect of RPS on the spiral arms of galaxies, we drew a sample of galaxies by visual inspection from the catalogue of galaxies in the GASP survey (described in Section 1). The GASP observations were reduced and processed as explained in GASP I (Poggianti et al. 2017b). In short, each galaxy was corrected for Milky Way dust reddening using dust values from NASA/IRSA (Infrared Science Archive) and correcting internal dust extinction using the Balmer decrement and the Cardelli, Clayton \& Mathis (1989) extinction law. Stellar absorption was taken into account using the spectral fitting code SINOPSIS (Fritz et al. 2017) to estimate the contribution from the stellar component, which was then subtracted from each spaxel. The data were then analysed using KUBEVIZ (Fumagalli et al. 2014; Fossati et al. 2018) as detailed in Bellhouse et al. (2017). KUBEVIZ fits a series of selected emission lines on a spaxel-by-spaxel basis with one or two Gaussian component profiles, allowing resolved analysis of the distribution and kinematics of the major emission lines in the observation to be carried out. We used KUBEVIZ on datacubes after smoothing with a $5 \times 5$ kernel, with the redshift set to the mean redshift for each galaxy and noisecube errors scaled to give a reduced $\chi^{2}=1$. The resulting fits to the emission lines across each galaxy gave the flux and velocity measurements used in this analysis. The stellar velocities for each galaxy were calculated using the absorption lines with the PPXF code (Cappellari \& Emsellem 2004) as described in Poggianti et al. (2017b). The sample of unwinding galaxies, i.e. galaxies that exhibit visual evidence of unwinding spiral arms, was selected from the full GASP catalogue of 94 jellyfish galaxies by visual inspection of the white-light MUSE images along with the $\mathrm{H} \alpha$ maps created using KUBEVIZ.

The visual selection was carried out with the aim of singling out objects with the following characteristics:

(i) Disc morphology (excluding mergers, rings, irregular galaxies, etc.).

(ii) Orientation close to face-on (axial ratio $>0.5$ ). This criterion ensures that the state of the spiral arms can be clearly inspected, which is not the case in edge-on galaxies.

(iii) Clear signatures of loose spiral arms, as judged from visual inspection of the $\mathrm{H} \alpha$ maps and white-light images.

Out of 12 face-on observed galaxies undergoing RPS with clear spiral arms in the full GASP sample, 11 met our criteria and exhibited signs of possible unwinding. The high incidence indicates that the effect could be prevalent in stripped galaxies. The one galaxy inclined face-on to the observer not showing the unwinding effect is galaxy JO128 that is located towards its cluster outskirts and belongs to the control sample of non-stripped galaxies given in Vulcani et al. (2018) due to the low star formation measured in the galaxy's tails.
In order to rule out gravitational interactions within the sample, a companion search was carried out using the photometric and spectroscopic data from OmegaWINGS. Criteria for the search were as follows:

(i) Photometric companions with $V$-band magnitude $<20$, at least 3 mag deeper than the faintest galaxy in the sample and within $20 \times$ $R_{\mathrm{e}}$ of the galaxy.

(ii) Spectroscopic companions with distance $<20 \times R_{\mathrm{e}}$.

Using these criteria, 7/11 galaxies have no discernible companions that could be a source of gravitational influence. The four galaxies with possible neighbours are: (1) JO201, whose neighbours are $>10 \times R_{\mathrm{e}}$ and two of which have large peculiar velocities; (2) JO194, with one neighbour at least 5 mag fainter than itself; (3) JO69, with a more compelling companion $57 \mathrm{kpc}$ from the galaxy, with similar velocity and magnitude; and (4) JO200, with three spectroscopic companions, all 3 mag fainter and outside $10 \times R_{\mathrm{e}}$.

Out of these, JO69 is the most likely to be affected by gravitational influence, while the companions of the other three galaxies are unlikely to be massive enough or close enough to cause any significant disturbance.

The final sample of unwinding spiral galaxies is listed in Table 1 and shown in Fig. 1. Additionally to the unwinding sample, we selected a control sample of galaxies from the GASP catalogue (see Vulcani et al. 2019, for the full GASP control sample). The selected control galaxies are not undergoing stripping and have faceon orientations, disc morphologies, and clear spiral arms as with the unwinding sample. The six control galaxies are also presented in Table 1. We note that two of the control sample galaxies, namely P63661 and P95080, are suspected to be experiencing cosmic web enhancement (Vulcani et al. 2019), but our results do not suggest that this is affecting the morphology of these galaxies.

\subsection{Morphologies}

As Fig. 1 shows, the effect of unwinding spiral arms is most visible in the $\mathrm{H} \alpha$ emission (not in the stellar light). Notable examples of unwinding spiral arms include $\mathrm{JO} 201$, which in the $\mathrm{H} \alpha$ map shows an extremely extended arc of stripped material to the east of the galaxy in Fig. 1, far outside the galaxy disc. JO200 shows a generally uniform spiral morphology, with three spiral arms extending below the disc in the image. While some galaxies exhibit asymmetric unwinding patterns in a distinct tail, JO85 and JO93 show an unwinding pattern most of the way around the disc. It is possible that the azimuthal extent of the unwinding feature around the galaxy disc may be proportional to the inclination of the galaxy with respect to the ICM; i.e. tighter tails are observed in galaxies moving edge-on into the wind, which unwind only on their trailing edges, whereas broader unwinding tails may indicate face-on stripped galaxies, whose tails originate from further around the disc.

All of the galaxies in the sample have generally undisturbed inner discs, which further diminishes the likelihood of any gravitational influence. In addition to the unwinding arms, all of the galaxies in the sample exhibit at least some other signatures of RPS, including extended extraplanar material, trailing clumps of star formation, and compression of the leading edge of the disc.

\subsection{Kinematics}

Fig. 2 compares the ionized gas kinematics on the left-hand panels with the stellar velocities on the right, for two example galaxies (JO85 and JO200). These galaxies were chosen in particular as archetypal 
Table 1. Sample of GASP jellyfish galaxies with unwinding spiral arms.

\begin{tabular}{|c|c|c|c|c|c|c|c|}
\hline \multicolumn{8}{|c|}{ Stripped galaxies } \\
\hline Galaxy & Cluster & $\begin{array}{l}\text { R.A. } \\
\text { (J2000) }\end{array}$ & $\begin{array}{l}\text { Decl. } \\
\text { (J2000) }\end{array}$ & $\begin{array}{c}M_{* \text {,disc }} \\
\left(10^{10} \mathrm{M}_{\odot}\right)\end{array}$ & Inclination & $\begin{array}{l}\text { Angle to } \\
\text { LOS motion }\end{array}$ & $\begin{array}{c}\text { Close to face-on } \\
\text { stripping }\end{array}$ \\
\hline JO201 & A85 & $00: 41: 30.29$ & $-09: 15: 45.900$ & $6.2 \pm 0.8$ & $41.4^{\circ}$ & $13^{\circ+21}$ & $\checkmark$ \\
\hline JO93 & A2593 & $23: 23: 11.74$ & $+14: 54: 05.013$ & $3.5 \pm 0.5$ & $25.0^{\circ}$ & $7_{-3}^{\circ+7}$ & $\checkmark$ \\
\hline JO194 & A4059 & 23:57:00.68 & $-34: 40: 50.117$ & $15.0 \pm 3$ & $38.3^{\circ}$ & $8^{\circ+16}$ & $\checkmark$ \\
\hline JO200 & A85 & 00:42:05.03 & $-09: 32: 03.841$ & $7 \pm 1$ & $45.3^{\circ}$ & $34_{-14}^{\circ+15}$ & - \\
\hline JO175 & A3716 & 20:51:17.60 & $-52: 49: 21.825$ & $3.2 \pm 0.5$ & $43.2^{\circ}$ & $61_{-15}^{\circ}$ & - \\
\hline JO47 & A168 & $01: 15: 57.67$ & $+00: 41: 35.938$ & $0.40 \pm 0.06$ & $44.7^{\circ}$ & $21_{-10}^{\circ}$ & $\checkmark$ \\
\hline JO49 & A168 & $01: 14: 43.85$ & $+00: 17: 10.091$ & $4.8 \pm 0.6$ & $53.6^{\circ}$ & $86^{\circ+2}$ & - \\
\hline JO70 & A2399 & 21:56:04.07 & $-07: 19: 38.020$ & $2.9 \pm 0.6$ & $39.3^{\circ}$ & $85_{-5}^{\circ+3}$ & - \\
\hline JO69 & A2399 & $21: 57: 19.20$ & $-07: 46: 43.794$ & $0.8 \pm 0.2$ & $43.3^{\circ}$ & $26^{\circ}{ }_{-11}^{16}$ & $\checkmark$ \\
\hline JO85 & A2589 & $23: 24: 31.36$ & $+16: 52: 05.340$ & $4.6 \pm 0.9$ & $24.9^{\circ}$ & $30^{\circ}{ }_{-11}$ & $\checkmark$ \\
\hline JW39 & A1668 & 13:04:07.71 & $+19: 12: 38.486$ & $17 \pm 3$ & $52.5^{\circ}$ & $31_{-15}^{\circ+15}$ & - \\
\hline \multicolumn{8}{|c|}{ Control galaxies } \\
\hline Galaxy & & $\begin{array}{l}\text { R.A. } \\
\text { (J2000) }\end{array}$ & $\begin{array}{c}\text { Decl. } \\
\text { (J2000) }\end{array}$ & $\begin{array}{c}M_{* \text {,disc }} \\
\left(10^{10} \mathrm{M}_{\odot}\right)\end{array}$ & Inclination & & \\
\hline P21734 & - & 11:31:07.90 & $-00: 08: 07.914$ & $6 \pm 1$ & $32.1^{\circ}$ & - & - \\
\hline P25500 & - & $11: 51: 36.28$ & $+00: 00: 01.929$ & $7 \pm 1$ & $50.0^{\circ}$ & - & - \\
\hline P48157 & - & $13: 36: 01.59$ & $+00: 15: 44.696$ & $3.9 \pm 0.8$ & $49.2^{\circ}$ & - & - \\
\hline P19482 & - & $11: 22: 31.25$ & $-00: 01: 01.601$ & $2.2 \pm 0.4$ & $54.9^{\circ}$ & - & - \\
\hline P63661 & - & $14: 32: 21.79$ & $+00: 10: 41.628$ & $2.1 \pm 0.3$ & $42.4^{\circ}$ & - & - \\
\hline P95080 & - & $13: 12: 08.70$ & $-00: 14: 20.510$ & $1.3 \pm 0.5$ & $43.1^{\circ}$ & - & - \\
\hline
\end{tabular}

face-on and edge-on stripped galaxies, respectively, to compare the effect of both scenarios. The remainder of the sample of unwinding galaxies is shown in the appendix (Fig. A1).

The stellar velocities in both galaxies are much smoother and show mostly undisturbed rotation. In the ionized gas of JO85, the kinematics are similar to the central region of the stellar disc, and significantly different around the edges. While the stellar disc velocity contours are smoother and straighter at the northern and southern edges of the disc, the gas kinematics contours bend up to the right at the northern edge of the disc. This is likely to result from JO85's mostly face-on interaction with the ICM (see Section 3), pushing the gas around the edges of the disc to higher line-of-sight velocities. In the case of JO200, the gas velocities are similar to the stellar velocities, with some differences in the central gradient. The stripped ionized gas appears to continue rotating with similar velocity to the galaxy disc.

The smooth stellar kinematics in the two examples and the rest of the sample shown in Fig. A1, combined with the stellar morphology versus $\mathrm{H} \alpha$, confirm that the disturbances experienced by these galaxies are hydrodynamical in nature, as gravitational interactions would influence both the gas and stellar kinematics.

\section{GALAXY ORBITS}

In order to explore the orbits of the galaxies in the sample and the inclination angles between the galaxies and their directions of motion through the ICM, we looked at the morphologies of galaxies and their positions in projected phase space.

The location of the sample of unwinding galaxies across projected position versus velocity phase space is a useful parameter in this analysis, as this diagram can reveal the orbital histories of the cluster galaxies (see Jaffé et al. 2018). Fig. 3 shows the phase-space distribution of the unwinding-spiral jellyfish sample compared with the overall population of cluster galaxies from WINGS/OmegaWINGS.
The galaxies have been plotted as thumbnail images centred on their corresponding locations in phase space. For each galaxy, the $\mathrm{H} \alpha$ map is plotted in red over the white-light image. The thumbnail images have been rotated such that the BCG direction is always to the left, parallel to the $x$-axis.

Almost all the galaxies in our sample have negative peculiar velocities that indicate they are moving towards the observer. In particular, there are several galaxies (most notably JO201, JO194, and JO85) occupying the lower envelope of the trumpet-shape distribution of galaxies in phase space close to the cluster centre, which is an indication that they could be crossing the dense core of the ICM at high speeds on radial orbits along our line of sight on first infall (see Bellhouse et al. 2017; Jaffé et al. 2018). Only one galaxy has a comparatively high positive velocity at a similar clustercentric distance (JW39), which indicates that it is moving away from the observer.

According to the phase-space diagram, the unwinding sample does not occupy a single, specific region of phase space, but none are located in the virialized region of phase space (low velocity, low projected radii).

The grey shaded region and grey lines indicate the probable angle of a galaxy's velocity between line of sight and plane of the sky (where $0^{\circ}$ corresponds to motion exclusively along the line of sight and $90^{\circ}$ to exclusively on the plane of the sky), given its location in projected phase space. These were produced by analysing a sample of 42 simulated galaxy clusters $\left(M_{200}>1 \times 10^{14} \mathrm{M}_{\odot}\right)$. The $N$-body simulations used were run using GADGET (Springel et al. 2005) with a box length of the volume of $120 \mathrm{Mpc} h^{-1}$ box. Initial conditions were built for a redshift $=200$ using the publicly available package MUSIC (Hahn \& Abel 2011), applying a camb power spectrum (Lewis, Challinor \& Lasenby 2000), accurate to second order. The mass of a dark matter particle is $1.072 \mathrm{e} 9 \mathrm{M}_{\odot} h^{-1}$. Halo finding was conducted using ROCKSTAR (Behroozi, Wechsler \& Wu 2013) for all haloes down to $1 \times 10^{11} \mathrm{M}_{\odot} h^{-1}$. Each simulated cluster was observed from 50 random lines of sight and stacked to produce 


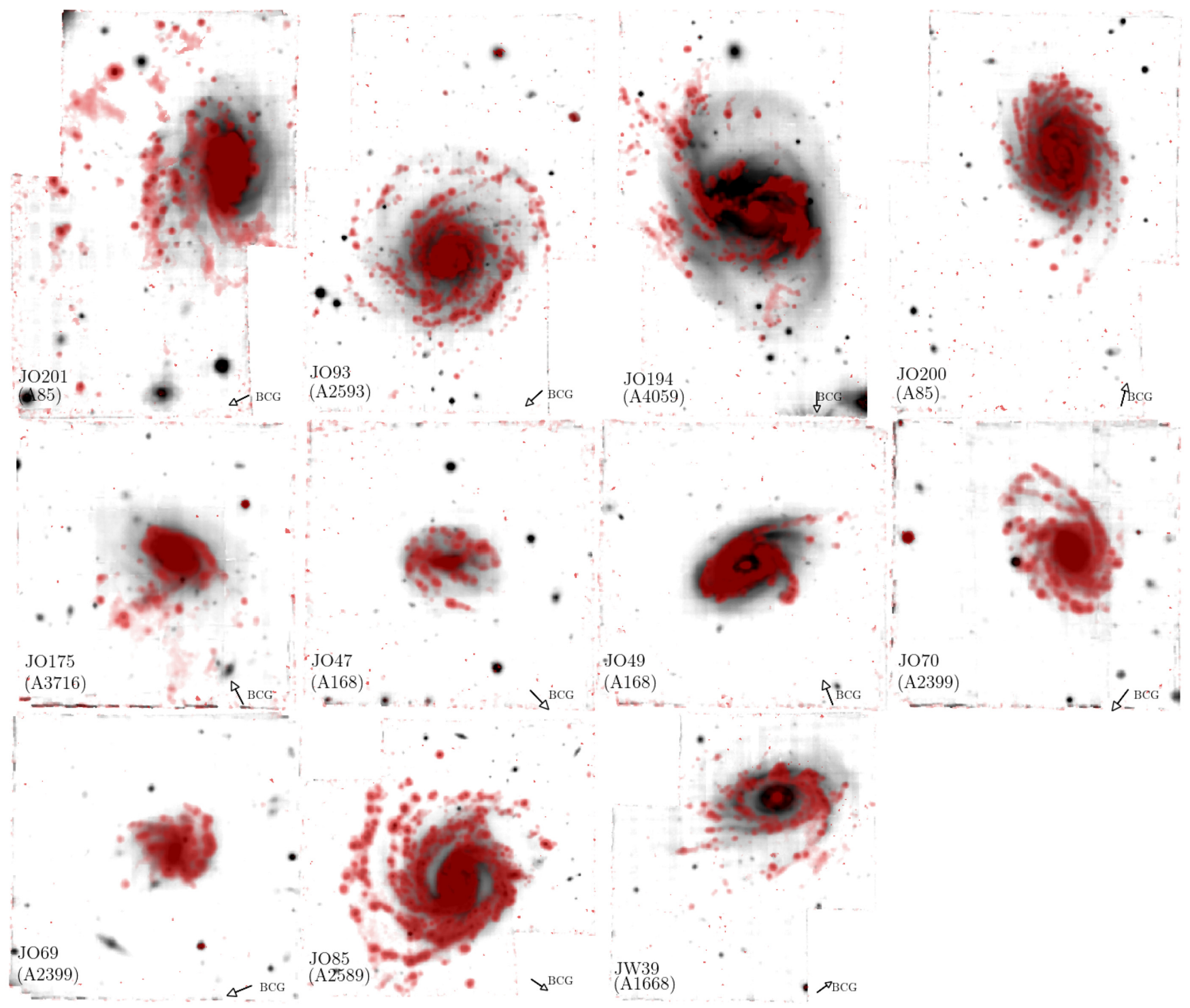

Figure 1. The unwinding galaxy sample, showing inverted white-light images of each galaxy with the $\mathrm{H} \alpha$ emission-line flux overlaid in red. The figure shows the red $\mathrm{H} \alpha$ tails extending far beyond the discs in some cases, while maintaining a clear spiral arm pattern. The white-light images generally show little to no unwinding pattern, which is expected to only manifest itself in the stripped gas and young stellar population.

a phase-space diagram of normalized projected radius and relative velocity. For each simulated cluster galaxy, the angle between its velocity vector and the line-of-sight axis was measured, and across the grid of phase space, these values were binned to give the median velocity angle for any given location. The galaxies in our observed sample were then assigned the velocity angle corresponding to their measured location in phase space. Further details of the simulations and method will be presented in an upcoming dedicated paper (Smith et al., in preparation).

Obtaining an estimate of the velocity angle for each galaxy allows us to more reliably predict whether a given galaxy is moving mostly along the line of sight or across the plane of the sky. For a galaxy viewed close to face-on, this yields the inclination of the galaxy with respect to the ICM wind, and therefore a measure of whether the galaxy is experiencing face-on or edge-on RPS. Since many galaxies in our sample are not viewed fully face-on, it is hard to predict the true wind angle due to uncertainties in the alignment of the galaxy's observed inclination and the true direction of motion along the plane of the sky. We can, however, use this as an indication of the likelihood of face-on stripping, which is more easily constrained. Galaxies viewed face-on and located outside the grey shaded area are therefore most likely to be experiencing face-on stripping. The values of the predicted angle to LOS (line of sight) motion are given in Table 1, as well as the likelihood that the velocity is within $30^{\circ}$ of the line of sight.

Out of the sample of 11 unwinding galaxies, 6 galaxies, namely JO201, JO93, JO194, JO85, JO69, and JO47, are all outside the grey region in phase space, and are moving within $30^{\circ}$ of the LOS. Coupled with their low inclinations, this suggests that these galaxies may be moving mostly face-on with respect to the ICM. The remaining five galaxies are likely to be undergoing either mostly edge-on stripping or a combination of the two.

The thumbnail images in the diagram give an indication of the projected tail directions of each of the unwinding galaxies with respect to the cluster centre. The general orientation of the tail towards the left or right (towards or away from the BCG, respectively) can indicate a galaxy's progression through infall, as the tail will be pointed away from the cluster centre up until pericentric passage and towards the cluster centre after pericentre; however, for the galaxies moving along 

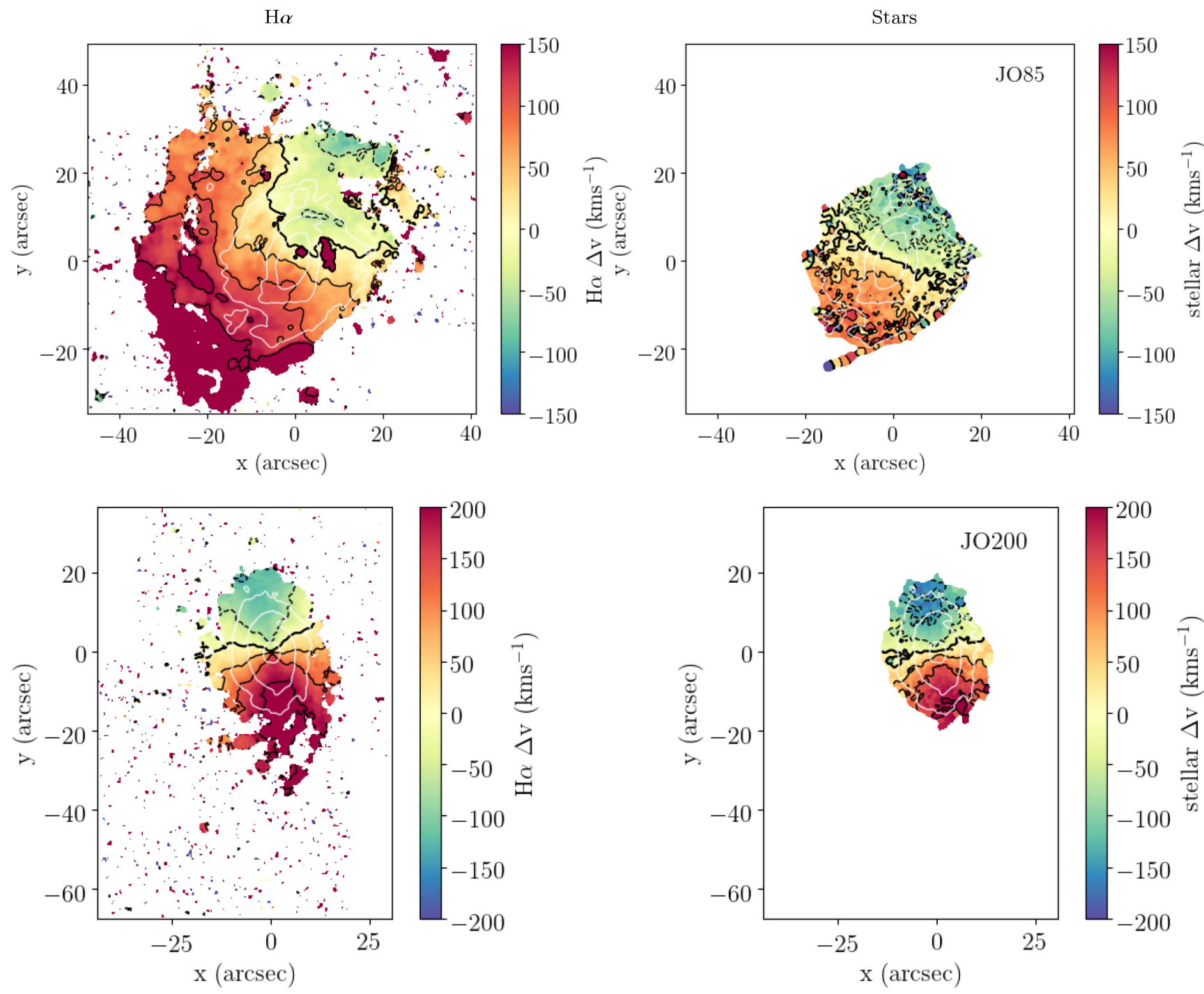

Figure 2. Comparison of kinematics maps in JO85 and JO200. Black lines indicate iso-velocity contours on the corresponding maps. The left-hand panels show the ionized gas velocity maps for each galaxy measured from emission-line kinematics. The right-hand panels show the stellar velocity maps measured using PPXF. Top, JO85: The stellar velocity contours exhibit a 'U'-shaped pattern at the upper and lower edges of the disc; in contrast, the gas kinematics follow concentric contours outwards from the upper edge of the disc. The gas kinematics show a slight systematic offset to the stellar kinematics as seen by the shift of the zero contour from the centre of the galaxy disc. Bottom, JO200: The stellar and gas velocities are similar in general, with some differences in the central gradient.

the line of sight, this is less well defined. The galaxies with more asymmetric tails, which lie to one side of the disc, are generally found within the grey shaded region, where they are likely to be experiencing some degree of edge-on stripping. Meanwhile, the galaxies with shorter, broader tails, which in some cases appear to be more symmetrically unwinding, are generally located within the 'face-on' stripping region at high LOS velocities, outside the grey region.

\section{SPIRAL ARMS}

\subsection{Identifying and characterizing spiral structure}

In order to analyse the extent of the unwinding spiral arm patterns in the galaxy sample, we produced maps of the $\mathrm{H} \alpha$-emitting ionized gas, reprojected on to polar space in terms of radial distance from the centre of the galaxy versus azimuthal angle around the disc.
The tightness of a spiral arm of a galaxy is measured by the 'pitch angle', which refers to the angle between a spiral arm and the tangent to the circle on the plane of the disc, where zero lies along the tangent and $90^{\circ}$ lies along the normal. More tightly wound spiral arms have lower pitch angles while steeper, more loosely wound spiral arms have higher pitch angles. We thus measured the pitch angles to quantify the unwinding by comparing inner and outer regions of the galaxy discs, in both the stripped and control sample galaxies. Higher pitch angles in the outer regions of the disc compared with the inner disc would reveal that the spiral arms are effectively being unwound.

We chose to implement a manual method of measuring the pitch angles of the spiral arms in this analysis. In particular, this is because these galaxies, by selection, show very disturbed morphologies that are not easily fitted using an automated process. In some cases, particularly $\mathrm{JO} 201$, the stripped material is so far removed from the galaxy that it barely qualifies as a conventional 'spiral arm', but its shape and alignment certainly indicate that it is likely to have 

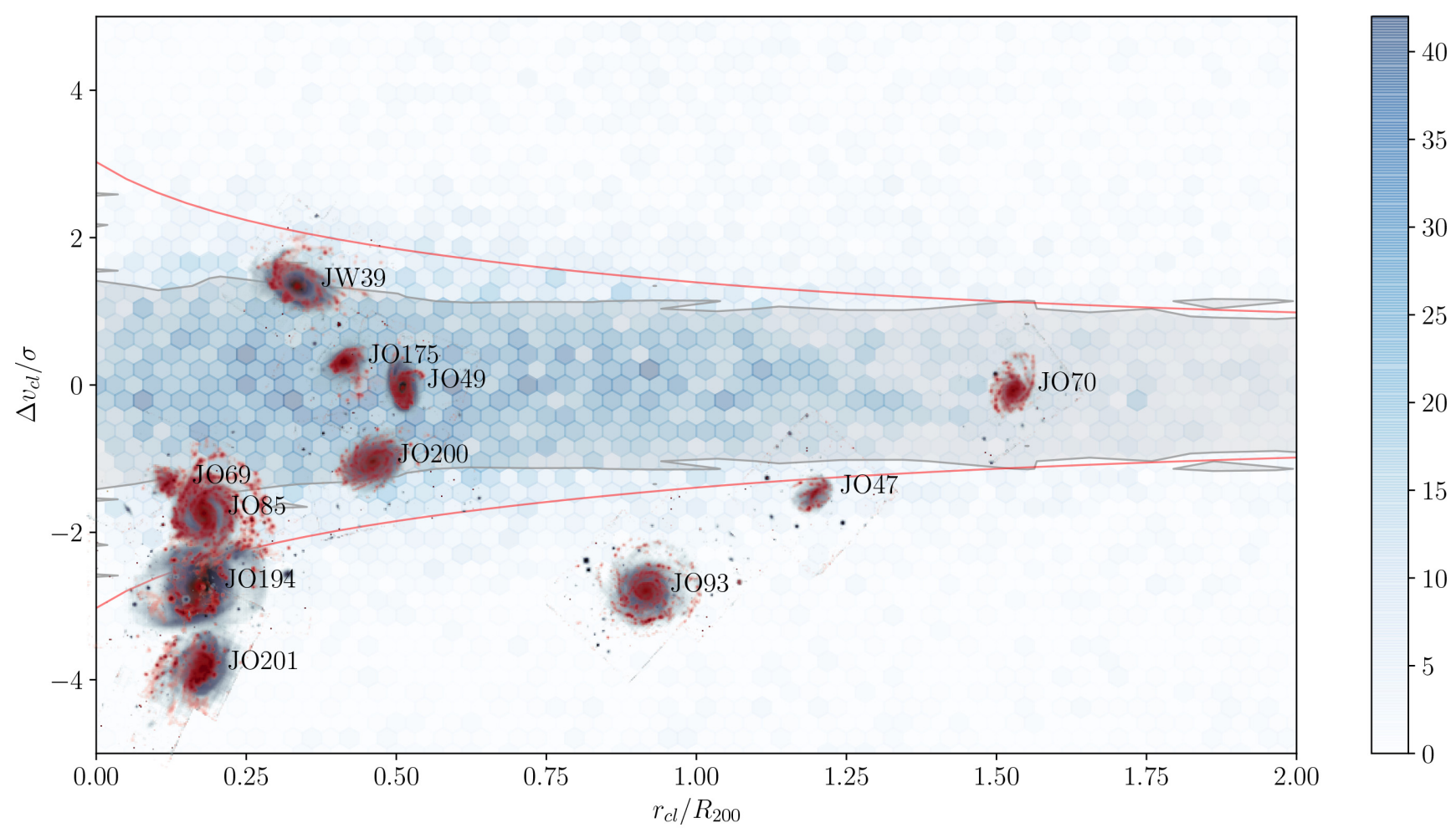

Figure 3. Projected position versus velocity phase-space diagram of the unwinding sample, showing thumbnail images of each galaxy (colours as in Fig. 1). Each thumbnail is rotated so that the BCG direction is to the left. The sample of all omegaWINGS (Gullieuszik et al. 2015; Moretti et al. 2017) galaxies is shown in the background as the blue hex plot and the solid red lines indicate escape velocity curves in a NFW profile as in Jaffé et al. (2018). The grey shaded area encloses the region in which the mean velocity angle is greater than $30^{\circ}$ to the line of sight. Galaxies outside this region are most likely to be moving mostly towards or away from the observer.

originated from one. In other cases, the spiral arms have been greatly extended by stripping to one side of their galaxy, and compressed or removed on the other. In order to make use of these extreme cases, a manual method allowed more fine-tunable selection of the material used in defining each spiral arm, ensuring more accurate reproduction of the local pitch angles. Since the sample of unwinding galaxies shown here is fairly small, a manual method of fitting the spiral arms is also within the realm of practicality.

We use the $\mathrm{H} \alpha$ emission, as it traces the distribution of the star formation activity that is increased in the denser regions of gas and dust of the spiral pattern. The emission maps were taken directly from the KUBEVIZ fits. In order to carry out the reprojection, each galaxy was corrected for inclination according to the axial ratios measured in Franchetto et al. (2020), by scaling the distances along the dimension of the kinematic minor axis. Each spaxel was then reprojected on to polar coordinate space according to its radial distance and azimuthal position. The reprojection was carried out by calculating the position of each corner of each spaxel and transforming into the reprojected polar space, in order to preserve the extent of each spaxel in the new space, particularly in regions close to the galaxy centre where individual spaxels cover a large range of azimuthal angles. Fig. 4 shows example figures for JO85 and JO200 (same galaxies shown in Fig. 9) where the disc of the galaxy is displayed 'unwrapped' in the left-hand panel, alongside the original galaxy on the right. Logarithmic spiral arms with constant pitch angle in the galaxy appear as lines on the left-hand side, with the gradient of the lines indicating the pitch angle. A galaxy from the non-stripping control sample, P25500, is shown in Fig. 5 for comparison.
Straight lines were aligned with the spiral arms on the azimuthal figure by eye, and the reverse of the reprojection method was used to show these on the original galaxy, highlighting the arm locations.

In Fig. 4, the unwinding effect manifests itself as a steepening of the pitch angle in the stripped material. In most of the strongly unwinding galaxies, the steepening occurs not gradually but sharply, with distinctly differing pitch angles between the disc and stripped material; however, this steepening occurs at a different point for each spiral arm.

The pitch angle of each spiral arm was taken as the mean value of four measurements, independently repeated. This was then averaged between all spiral arms to obtain the global mean pitch angle within the disc (defined here as having average radii within $2 \times$ the effective radius and within the stellar continuum contours) and in the outer disc, for each galaxy.

The effective radii were calculated for each galaxy by analysing the azimuthally averaged surface brightness profile in $I$-band images generated from the MUSE data (Franchetto et al. 2020).

The pitch angles were also measured for the stellar continuum emission using the same method (not shown here). For the unwinding sample, these were all in agreement with the inner disc $\mathrm{H} \alpha$ pitch angles to within $10^{\circ}$, with an average offset of $4.8^{\circ}$, indicating that the inner spiral arm pitch angle is a reasonable measure of the 'undisturbed' pitch angle.

In the case of JO85, the spiral arms have a mean pitch angle of $17.3^{\circ}$ in the disc, which increases to around $23.0^{\circ}$ in the unwound tails. For JO200, the spiral arms inside and outside two effective radii have mean values of $22.0^{\circ}$ and $30.5^{\circ}$, respectively. In contrast, the 

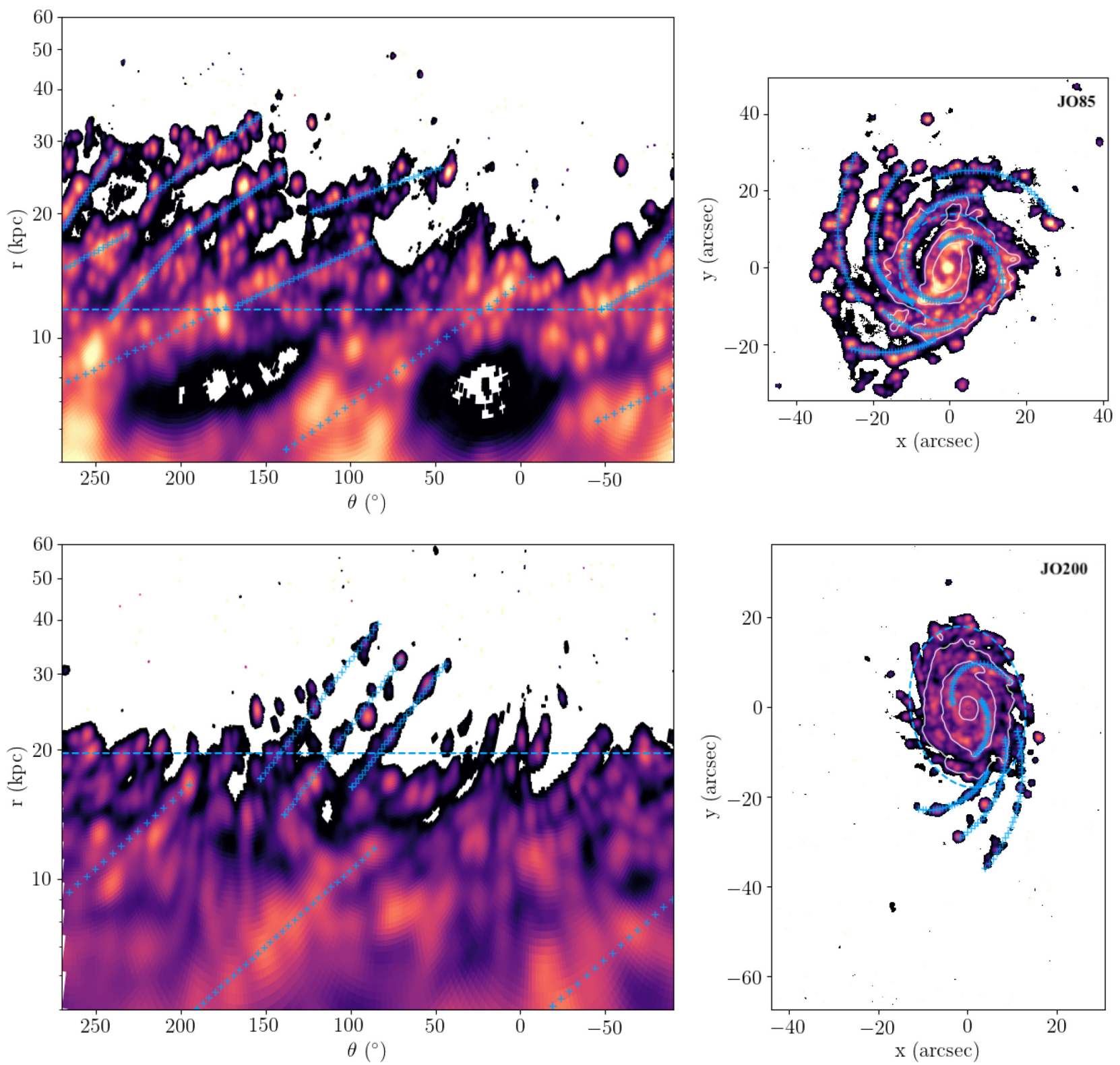

Figure 4. Azimuthal plots of $\mathrm{H} \alpha$ for the 'unwrapped' disc of JO85 (top) and JO200 (bottom), showing radial projected distance from the centre in logarithmic scale against azimuthal angle in the left-hand panels, alongside the original galaxy discs on the right-hand panels. The white contours on the right-hand panels denote stellar continuum isophotes. The blue dashed horizontal lines on the left-hand panels and the dashed blue ellipses on the right mark two effective radii on the disc. Lines of + symbols mark spiral arm patterns identified by eye on the unwrapped figure and are shown reprojected back on the original galaxy discs. The pitch angles of these spiral arms are shown along with their average radial distance in Fig. 6.

control galaxy shown here, $\mathrm{P} 25500$, has mean pitch angles of $24.8^{\circ}$ and $18.6^{\circ}$ inside and outside two effective radii, respectively.

The azimuthal plots of other galaxies, shown in Fig. C1, have average disc and tail spiral arm pitch angles shown in Table 2. Cases where spiral arms are not clear enough to measure an accurate pitch angle are indicated by question marks. Azimuthal projections of the simulated galaxies (see Section 5) were also produced using the same technique for comparison, and are shown in Fig. B1.

To measure and characterize the extent of 'unwinding' in the outer spiral arms, the pitch angles for all galaxies in the unwinding and control samples, as well as the simulated unwinding galaxies, are shown in Fig. 6 against their mean radial distance in effective radii. For each galaxy, the mean inner and outer spiral arm pitch angle and radius values are connected by grey lines for comparison. The left-hand panel of Fig. 6 shows the unwinding sample, while the right-hand panel shows the control galaxies. The outer spiral arms of the control galaxies extend much less far than the unwinding galaxies. The ranges of masses and redshifts of the control galaxies are the same as those of the stripped sample; therefore, the lack of spiral arms at higher radii in the control galaxies is due to the absence of only stripped material. It is clear from the figure that the control galaxies have much lower outer spiral arm pitch angles compared to the stripped galaxies, and the gradients between inner and outer pitch angles are generally low, indicating that the pitch angles do not vary with radius. For some galaxies in the stripped sample, the variation between inner and outer disc pitch angles is not 

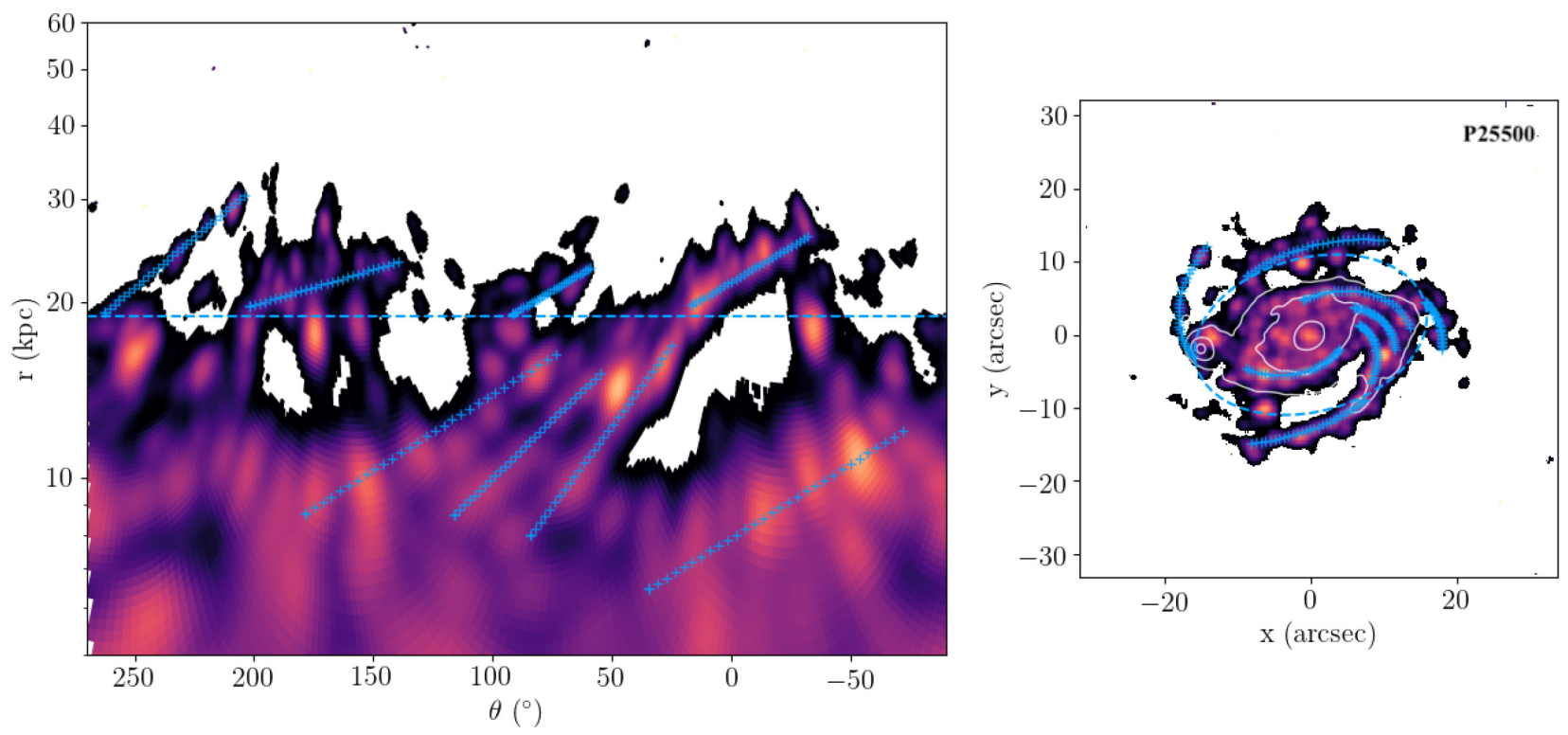

Figure 5. Plot of $\mathrm{H} \alpha$ for the 'unwrapped' disc of P25500, showing radial projected distance from the centre in logarithmic scale against azimuthal angle, alongside the original galaxy disc on the right. The white contours on the right-hand panel denote stellar continuum isophotes. The dashed blue line on the left-hand panel and the dashed blue ellipse on the right both indicate two effective radii on the disc. Lines of ' + ' symbols mark spiral arm patterns identified by eye on the unwrapped figure and are shown reprojected back on the original galaxy disc. The pitch angles of these spiral arms are shown along with their average radial distance in Fig. 6.

Table 2. Comparison of pitch angles in the discs and tails of unwinding GASP jellyfish galaxies. The numbers of inner and outer pitch angle measurements used in the analysis are listed. Values marked as ? are given where no clear spiral arms are visible.

\begin{tabular}{lcccc}
\hline $\begin{array}{l}\text { Stripped galaxies } \\
\text { Galaxy }\end{array}$ & $N_{\text {inner }}$ & $\alpha_{\text {inner }}\left({ }^{\circ}\right)$ & $N_{\text {outer }}$ & $\left.\alpha_{\text {outer }}{ }^{\circ}\right)$ \\
\hline JO201 & 0 & $?$ & 4 & 21.5 \\
JO93 & 4 & 11.1 & 5 & 21.9 \\
JO194 & 2 & 13.1 & 2 & 53.4 \\
JO200 & 2 & 22.0 & 3 & 30.5 \\
JO175 & 1 & 27.0 & 2 & 66.5 \\
JO47 & 0 & $?$ & 0 & $?$ \\
JO49 & 2 & 26.2 & 2 & 46.5 \\
JO70 & 2 & 21.3 & 2 & 26.4 \\
JO69 & 0 & $?$ & 0 & $?$ \\
JO85 & 4 & 17.3 & 4 & 23.0 \\
JW39 & 1 & 7.4 & 1 & 46.3 \\
Control galaxies & & & & \\
P21734 & 3 & 18.8 & 3 & 27.4 \\
P25500 & 4 & 24.8 & 4 & 18.6 \\
P48157 & 3 & 19.7 & 2 & 19.4 \\
P19482 & 2 & 26.9 & 2 & 25.6 \\
P63661 & 2 & 21.7 & 1 & 21.2 \\
P95080 & 2 & 14.6 & 2 & 15.6 \\
\hline
\end{tabular}

much greater than that in the control sample; however, in a couple of stronger cases, notably JO194, JW39, and JO175, the outer disc pitch angles can be more than double the inner disc pitch angles. It is important to consider that uncertainties in the inclination will affect these numbers. Furthermore, in the case of galaxies that are not fully edge-on stripped, any stripped material that lies extraplanar to the disc will be incorrectly deprojected for inclination; however, this should be addressed by taking the average pitch angle at all azimuthal positions. These results indicate that in some cases, the 'unwinding' can indeed be characterized by a measurable increase in pitch angle suggesting a loosening of the spiral arms. In other cases, we instead see only 'apparent' unwinding, whereby the effect may be the result of the spiral arms being stretched along the direction of stripping, giving the appearance of unwinding without altering the pitch angle.

\subsection{Gradients in stellar ages}

In order to indirectly estimate the time-scale involved in the unwinding effect, we constrained the spatial distribution of the stellar component, divided into bins of stellar age calculated using the SINOPSIS spectrophotometric fitting code (cf. Fritz et al. 2017). SINOPSIS finds the best-fitting combination of single stellar population spectra to the spectrum of each spaxel, including the pertinent emission lines, in order to estimate the contributions of stellar populations in four age bins (1) $5.7 \times 10^{9}-1.4 \times 10^{10} \mathrm{yr}$, (2) $5.7 \times 10^{8}-5.7 \times 10^{9} \mathrm{yr},(3)$ $2 \times 10^{7}-5.7 \times 10^{8} \mathrm{yr}$, and (4) $<2 \times 10^{7} \mathrm{yr}$ to the observed light.

Fig. 7 shows the white-light images of each galaxy, overlaid with the oldest stellar component $\left(5.7 \times 10^{9}-1.4 \times 10^{10}\right)$ in red and the youngest $\left(<2 \times 10^{7} \mathrm{yr}\right)$ in blue. The image reveals that, as expected for purely hydrodynamical disturbance, the older stellar component shown by the red contour line lies solely within each galaxy and appears undisturbed by the stripping. In contrast, the blue contours that trace the newly formed stars more closely follow the pattern of the unwinding spiral arms and extend much further $(\sim 20 \mathrm{kpc})$ outside the galaxy disc. This shows that the stars form out of the spiral arms after they have been stripped and unwound from the galaxy; the newly formed stars will be unaffected by subsequent stripping.

Highlighting this latter point more precisely, Fig. 8 shows the most drastic examples of spatial offsets between stellar age bins within the unwinding tails. In both figures, red shows the oldest stellar population $\left(5.7 \times 10^{9}-1.4 \times 10^{10} \mathrm{yr}\right)$, followed by orange $\left(5.7 \times 10^{8}-5.7 \times 10^{9} \mathrm{yr}\right)$ and green $\left(2 \times 10^{7}-5.7 \times 10^{8} \mathrm{yr}\right)$, with blue showing the youngest $\left(<2 \times 10^{7} \mathrm{yr}\right)$. In the upper left of JO194, the 
Unwinding Sample

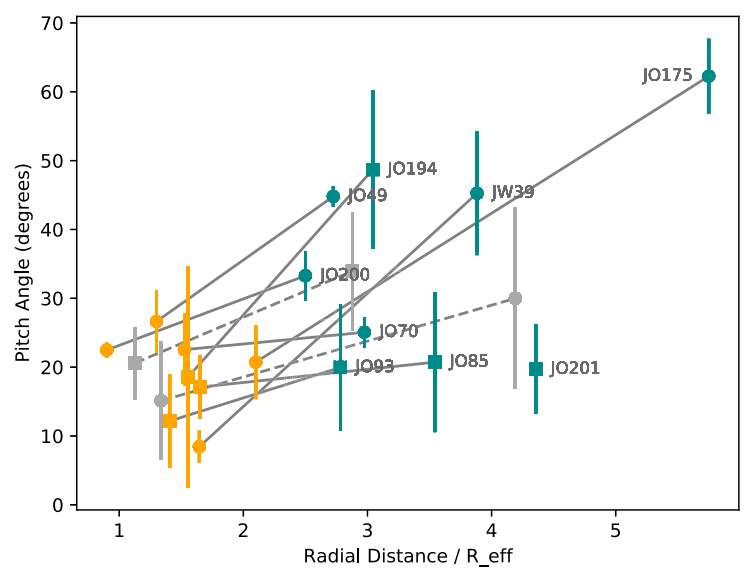

Control Sample

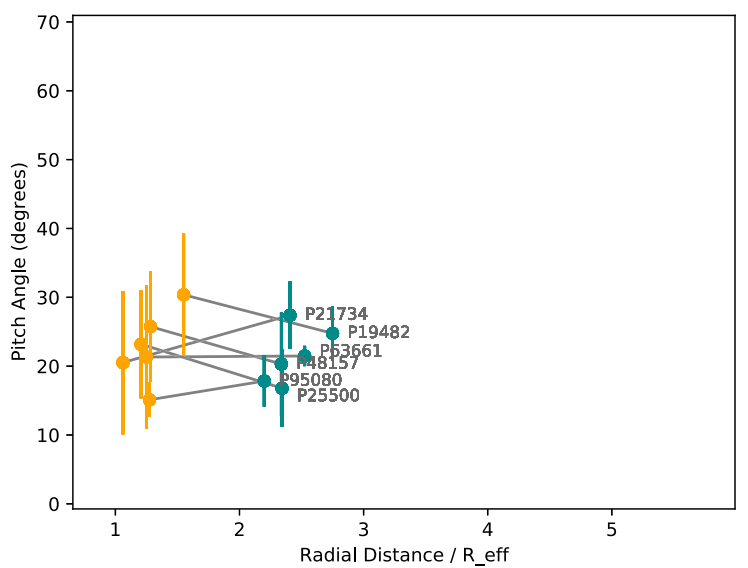

Figure 6. Measured average pitch angles for all galaxies in the unwinding sample (left) and control sample (right). 'Inner' spiral arms, marked in orange, are defined where the average radial distance is within $2 r_{\text {eff }}$ and the arm lies within the visible stellar continuum contours (examples shown in white in the right-hand panels of Fig. 4). Outer spiral arms are marked by teal points. Galaxies tagged as face-on interacting in Table 1 are shown as square markers. The values are connected by grey lines to indicate which points belong to the same galaxy, and errorbars mark the standard deviation between the measured pitch angles. The grey points connected by dashed lines indicate the simulated face-on and edge-on stripped galaxies, the azimuthal plots for which are shown in Fig. B1.

green and blue contours show a distinct spatial offset of around $5 \mathrm{kpc}$, which traces the motion of the unwinding arm. The green contour of stars that formed around $300 \mathrm{Myr}$ earlier is more tightly wound with the disc of the galaxy, while the blue contour of younger stars lies further extended from the disc, around $6^{\circ}$ steeper in pitch angle. A similar occurrence is visible in JO85 with the lower spiral arm of the image, with a less pronounced $4^{\circ}$ increase in pitch angle. This pattern captures the process of the spiral arm opening out and leaving a trail of newly formed stars in its wake. As the spiral arm material is stripped from the galaxy and begins to undergo star formation, the stars, as mentioned before, remain unaffected by subsequent ram pressure, resulting in an age gradient. In many ram-pressure stripped galaxies, such age gradients are observed along the knots of star formation (Sheen et al. 2017; Jáchym et al. 2019) as diffuse gas collapses along its motion trailing behind the galaxy (Yoshida et al. 2008; Kenney et al. 2014); here, the same effect reveals the outstretching of the spiral arm during the stripping process.

\section{SIMULATIONS}

To understand more precisely the process, or processes, by which hydrodynamical interactions can result in unwound spiral arms, we compare the observed morphology and velocity field of our sample to a set of high-resolution wind-tunnel simulations of an intermediate-mass spiral galaxy undergoing RPS. Previous works have shown that the inclination of the ICM wind to the galaxy disc affects the efficiency of stripping (Roediger \& Brüggen 2006; Jáchym et al. 2009), and have shown that unwinding can occur in certain circumstances (Schulz \& Struck 2001; Roediger et al. 2014; Steinhauser et al. 2016). In this study, we comprehensively analyse the evolution of the unwinding pattern over time for galaxies at different inclinations, comparing directly with the morphology and kinematics of the observed sample and features detected with MUSE. The simulations consider fixed inclination angles for the direction of the ram-pressure wind, face-on and edge-on with respect to the plane of the model's disc. In this way, we hope to better understand the role of inclination on the velocity field of the gas, as well as the effectiveness with which the spiral arms are unwound and the pattern of unwinding.

The model spiral galaxy we use has three main components of mass: a NFW dark matter halo, exponential disc of stars, and exponential disc of gas. Our model galaxy does not contain a bulge component. The dark matter halo mass of the galaxy is $M_{\text {total }}=3.2 \times 10^{11} \mathrm{M}_{\odot}$, with a virial radius of $140 \mathrm{kpc}$, and halo concentration $=12$, consisting of 5 million dark matter particles. The stellar disc has a total mass of $M_{*}=1 \times 10^{10} \mathrm{M}_{\odot}$, with a radial exponential scale length of $2.2 \mathrm{kpc}$, truncated at 4 scale lengths, initially consisting of 250 thousand star particles. The gas disc initially has a total mass of $M_{\text {gas }}=1.3 \times 10^{9} \mathrm{M}_{\odot}$, and a radial scale length of $3.7 \mathrm{kpc}$, truncated at 4 scale lengths, and is assumed solar metallicity. Initial conditions were built using the publically available initial-conditions set-up code DICE (Perret et al. 2014). Tests demonstrate that the initial conditions were very stable, and were evolved for $0.5 \mathrm{Gyr}$ in isolation to ensure that dynamical stability had been reached.

Simulations were carried out with the Adaptive Mesh Refinement code RAMSES (Teyssier 2002). The total volume was a box with a side length of $280 \mathrm{kpc}$, which fully encloses the virial radius of the dark matter halo. The adaptive mesh was allowed to refine according to the mass within a cell to a maximum refinement level $=14$, equivalent to a smallest cell size of $17 \mathrm{pc}$. In addition, the refinement level ensures that at least four Jeans lengths are resolved, down to the maximum level of refinement. The simulation considers a radiative-cooling treatment for solar-metallicity gas. Star formation occurs following a standard prescription of a Schmidt relation with a 3 per cent star formation efficiency for all gas above a critical density threshold of $0.1 \mathrm{~cm}^{-3}$. Supernova feedback is treated thermally, with an efficiency of 20 percent, and a yield of 0.1 . We confirm that, in isolation, our model disc lies on the star-forming main sequence.

Ram pressure was modelled using a wind-tunnel simulation set-up. A hot, fast-flowing, low-density, ICM gas is fed into the simulation box from one wall of the simulation box. We initialize the disc with a low constant wind to roughly imitate the outskirts of the cluster. Then, after reaching $1 \mathrm{Mpc}$ from the cluster centre, the density and inflow 

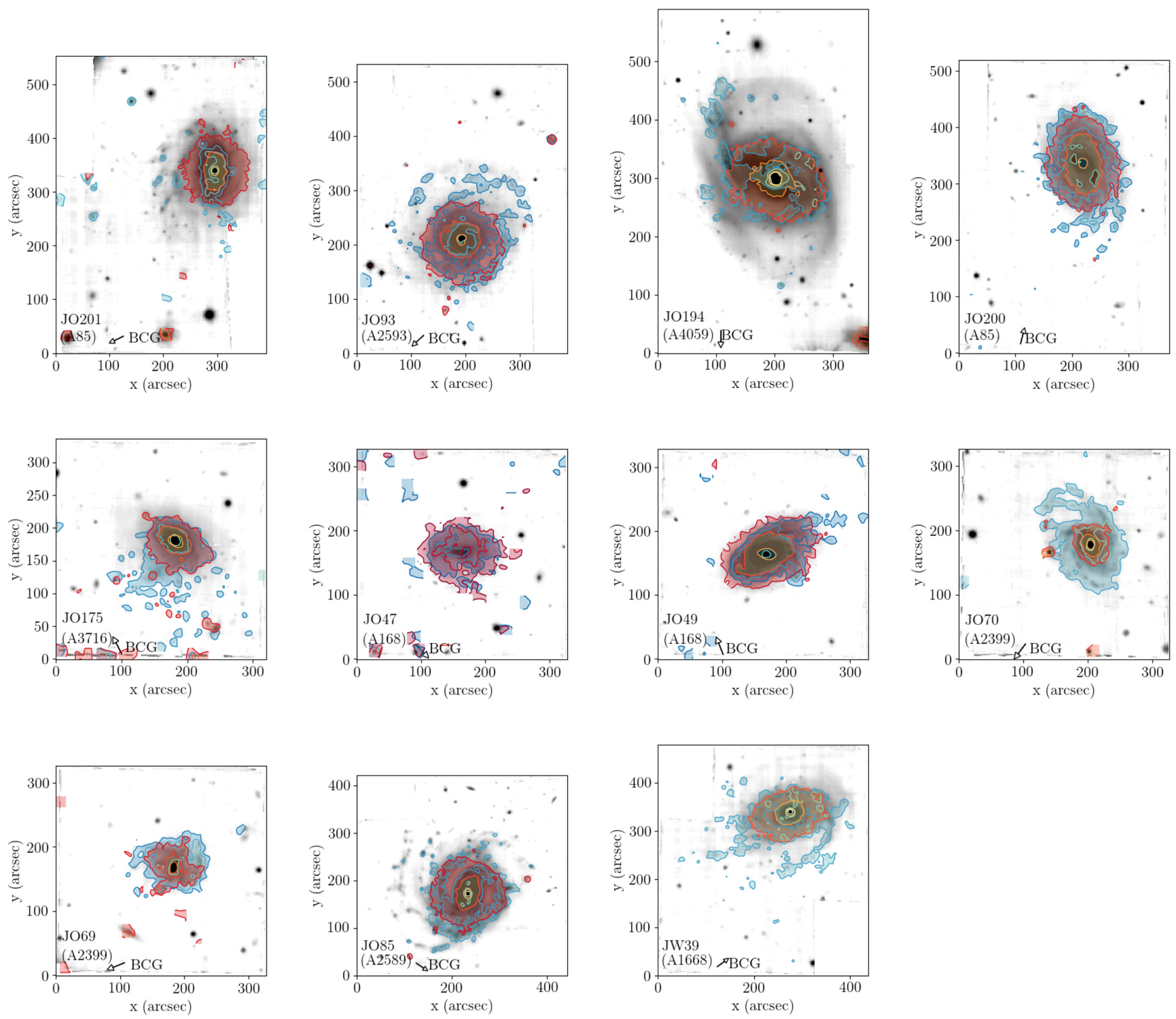

Figure 7. Unwinding galaxies shown in white light, overlaid with pairs of contours showing the oldest $5.7 \times 10^{9}-1.4 \times 10^{10} \mathrm{yr}($ red/orange) and youngest $<2 \times 10^{7} \mathrm{yr}$ (blue/light blue) stellar populations calculated using the SINOPSIS spectrophotometric code. The figures show that the older stellar population is generally constrained to the disc of the galaxy and is fairly undisturbed, while the younger population of stars extends much further into the tails and describes the extended spiral structure in the unwound arms.

velocity of the ICM begin to smoothly evolve with time, to mimic the changing ram pressure that a cluster spiral might experience when falling into a real cluster. To calculate the time evolving density, we assume a beta model for the ICM that is a rough approximation for the Virgo cluster (central density $=2.0 \times 10^{-26} \mathrm{~g} \mathrm{~cm}^{-3}, \beta=0.5$, core radius $=50 \mathrm{kpc}$ ). To calculate the time evolving wind speed, we calculate the orbital velocity of a galaxy moving through the cluster potential well, and assume that the inflow speed matches the orbital velocity. Under the assumption of hydrostatic equilibrium with a gas temperature of $4.7 \times 10^{7} \mathrm{~K}$, the potential well of the cluster is fully defined. Using a simple time-step integrator, we trace out the orbit of a galaxy falling in from $1 \mathrm{Mpc}$. We assume that the galaxy has an initial radial velocity component of $565 \mathrm{~km} \mathrm{~s}^{-1}$, and a tangential velocity component of $565 \mathrm{~km} \mathrm{~s}^{-1}$. This results in quite a plunging orbit reaching a pericentric distance of $340 \mathrm{kpc}$ from the cluster centre. During the infall, the orbital velocity increases from
$800 \mathrm{~km} \mathrm{~s}^{-1}$ and reaches a maximum velocity of $\sim 1600 \mathrm{~km} \mathrm{~s}^{-1}$ at pericentre, and we set the inflow speed of the gas in our wind-tunnel test as equal to the orbital velocity.

For each of the inclination angles considered, we conduct the simulation for at least $2.3 \mathrm{Gyr}$ in total. This is sufficient to pass the cluster pericentre, which occurs after approximately $1.7 \mathrm{Gyr}$. For this study, we find that the heavily truncated gas discs of those galaxies that have passed pericentre do not show features resembling the unwrapping we see in our observational sample. Therefore, we restrict our attention to the period between when the model discs enter the cluster and just before they reach pericentre.

Now we create 'observed' velocity maps of the simulated discs at various instants in time, as they approach the cluster pericentre for the first time. We consider instants when the galaxy is at a cluster centric radius of $r_{\mathrm{cl}}=0.92 \mathrm{Mpc}$ (in the cluster outskirts), $0.66 \mathrm{Mpc}$ (intermediate radius), and $0.35 \mathrm{Mpc}$ (just before pericentre). At each 

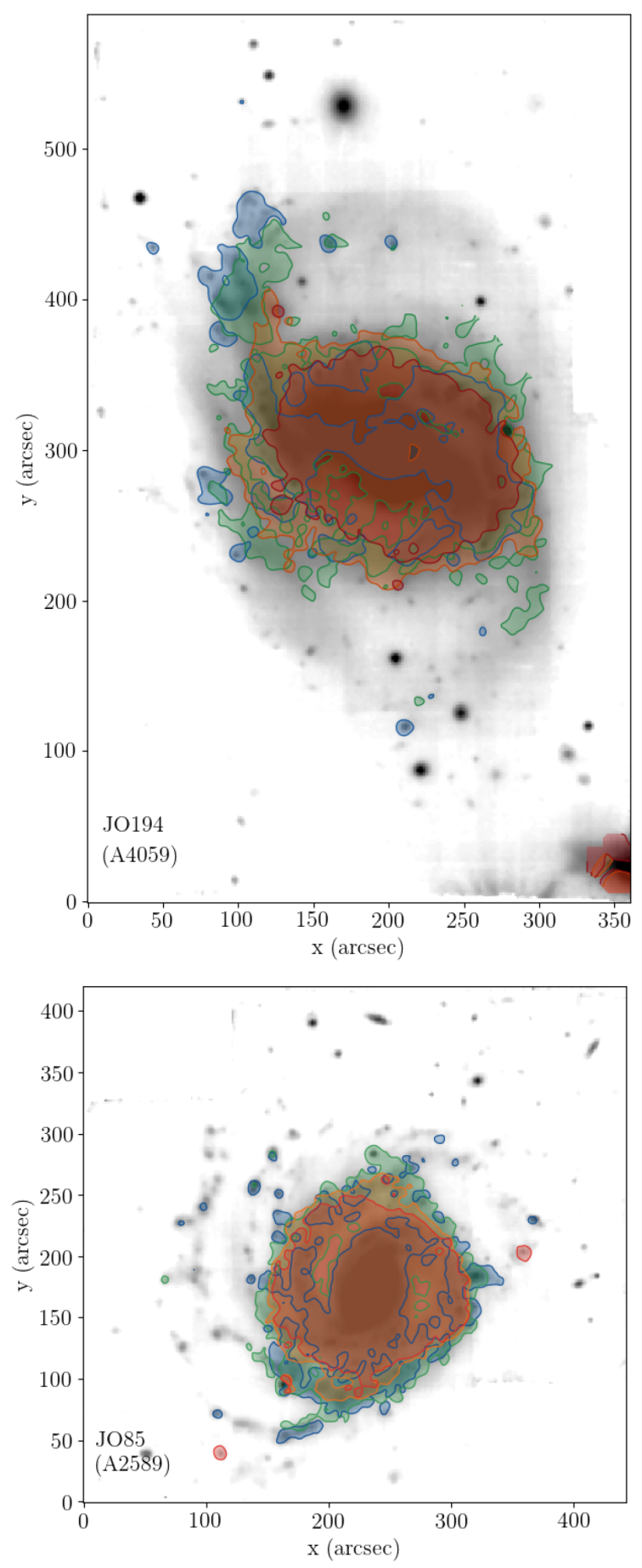

Figure 8. Unwinding galaxies shown in white light, overlaid with four stellar age bins. Red: $5.7 \times 10^{9}-1.4 \times 10^{10} \mathrm{yr}$, orange: $5.7 \times 10^{8}-5.7 \times 10^{9} \mathrm{yr}$, green: $2 \times 10^{7}-5.7 \times 10^{8} \mathrm{yr}$, and blue: $<2 \times 10^{7} \mathrm{yr}$. Top, JO194: The upper left arm shows that the youngest (blue) stellar population is much further unwound from the galaxy than the green stellar age bin, indicating that the stars form out of the unwinding gas cloud and remain in the position in which they formed. As the arm unwinds, it has left behind a trail of newly formed stars in its wake. Bottom, JO85: The lower spiral arm shows a similar spatial offset to JO194 between the two youngest stellar populations revealing the unwinding motion of the spiral arm as it leaves a trail of newly formed stars. of these instants, we produce maps by binning the gas properties along a given line of sight, considering similar resolution as in the MUSE observations. We produce maps for both disc inclinations. As we are comparing to $\mathrm{H} \alpha$ emission-line maps, we only consider gas above the star formation threshold in the simulation (i.e. with density $>0.1 \mathrm{~cm}^{-3}$ ) in our maps. To produce kinematics maps for comparison with the GASP observed galaxies, the data were transformed to different lines of sight and binned across the plane of observation to a similar resolution to MUSE, and then the mean velocity within each bin was taken to produce the velocity maps.

\subsection{Comparing observations with simulations}

JO200 and JO85 were selected as suitable galaxies for comparison with the simulation models, given the clarity of their disc and spiral arms, as well as strongly symmetric and asymmetric unwinding, respectively, as definitive cases for each. The masses of the observed galaxies do not match the masses of the simulated examples, but we are only concerned here about the pattern produced by the interaction.

The compared velocity fields of JO200 and the edge-on simulated galaxy are shown in Fig. 9. For ease of comparison between the two, the simulated galaxy was rotated by transforming the data to match the inclination and position angle of JO200, mimicking the observed line of sight of the galaxy. The mean particle velocities along each pixel 'bin' gave the kinematics maps shown in the figure.

While the absolute values of the rotation curve differ as a result of the different total masses of the real and simulated galaxies (JO200 $M_{*}=7 \times 10^{10}$, simulation $\left.M_{*}=1 \times 10^{10}\right)$, the gradient of the rotation is very similar in both cases. It is apparent from the figure that the velocity contours in the observed galaxy are more ' $U$ '-shaped at the most extreme ends of the rotation curve, while the simulated galaxy exhibits a more uniform rotation. The spiral arms in both the simulated and observed galaxies show a similar amount of unwinding with similar pitch angles (JO200: inner $=22.0$ outer $=30.5$, Edge-on sim: inner $=15.6$ outer $=30.5$ ) in the unwound tails at the lower edge of the galaxies. The resemblance suggests that the unwinding pattern of JO200 may be well described by a nearly edge-on stripping scenario.

The velocity pattern in JO85 shows similarities to the face-on stripping simulated galaxy, particularly in the curvature of the zero contour where the top end bends to the right as the stripping begins to skew the velocities at the edge of the disc. The similarities suggest that the JO85 is experiencing close to face-on motion, but differences and asymmetries suggest that some component of edge-on motion is also present. The kinematic patterns that evidence face-on and edgeon interactions will be further explored in the simulations in the next section.

\subsection{Tracing unwinding throughout infall}

We further studied the mechanisms behind the process of unwinding by analysing the kinematics and shape of the simulated galaxies over several time-steps throughout the infall process. Snapshots were taken of the edge-on and face-on galaxies during three stages (81, 416, and $789 \mathrm{Myr}$ ) throughout the infall process. Additionally, a snapshot taken prior to infall is shown for the edge-on galaxy. These snapshots were binned to a grid of pixels at a similar resolution to the observations and, in the case of edge-on, analysed in two 'observed' orientations: (1) the galaxy is falling partially along the line of sight, and (2) the galaxy is falling across the plane of the sky. The snapshots of the two cases are shown in Figs 10 and 11, respectively. 

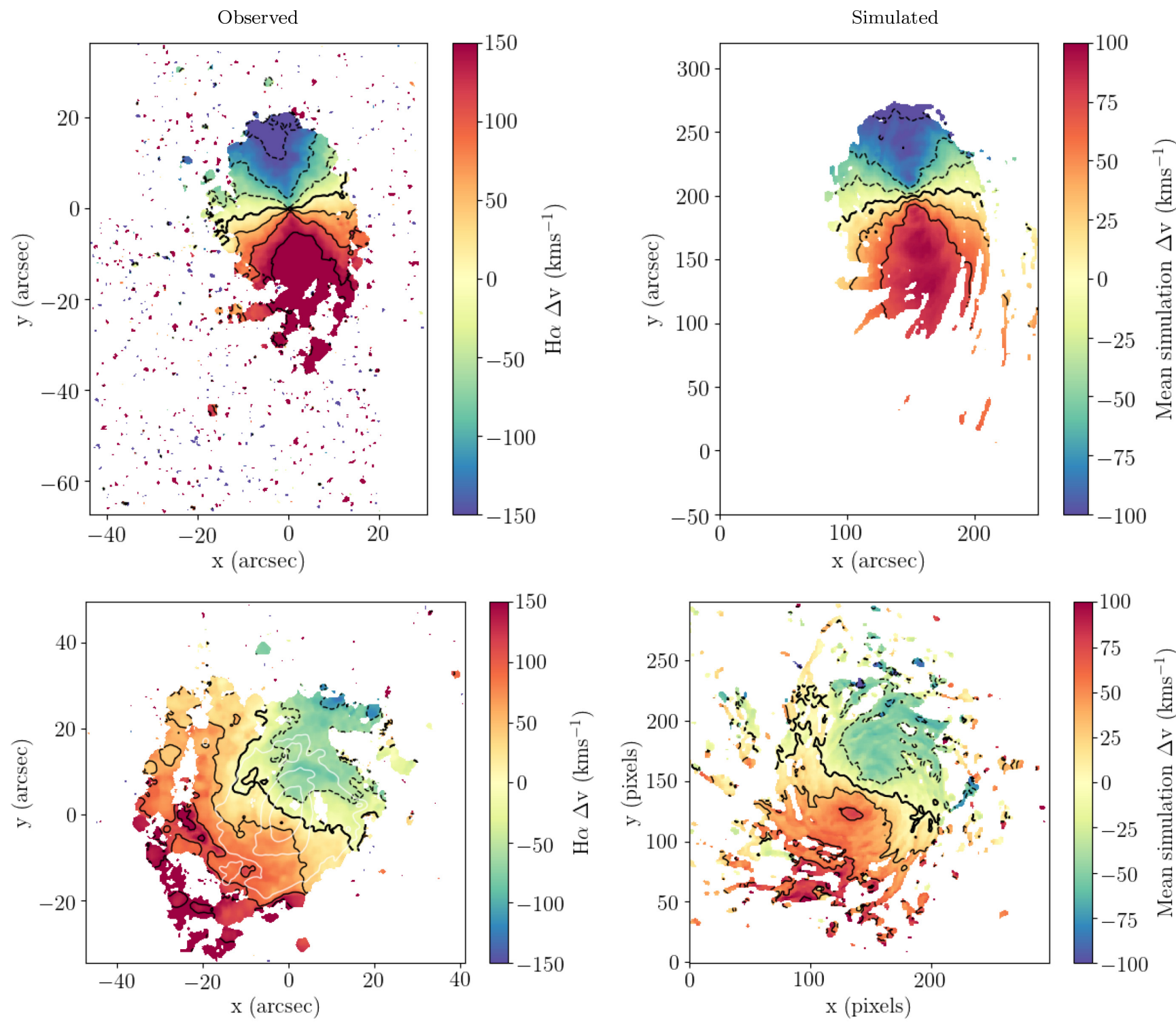

Figure 9. Top left: Kinematics map of JO200 measured using $\mathrm{H} \alpha$ emission-line velocities from KUBEvIz. Top right: Kinematics map of 'observed' simulated galaxy undergoing edge-on stripping, produced as described in Section 5.1. The simulated unwinding galaxy is rotated to match the inclination and position angle of JO200, and matches the unwinding pattern and kinematics fairly well, suggesting that JO200 is well described by an edge-on stripping scenario. Bottom left: Kinematics map of JO85 measured using $\mathrm{H} \alpha$ emission-line velocities from KUBEVIZ. Bottom right: Kinematics map of 'observed' simulated galaxy undergoing face-on stripping. The simulated unwinding galaxy is rotated to match the inclination and position angle of JO85. The overall similar kinematics suggest that JO85 may be undergoing close to edge-on stripping, but differences and asymmetries suggest that some component of edge-on motion is also present.

In the line-of-sight case, Fig. 10, it can be seen that the gas on the advancing edge of the galaxy, the right hand edge in the figure, is slowed by the ram pressure as it rotates into the wind. As the wind slows the gas, it causes it to build up as well as fall to higher orbits, producing a large asymmetry in the second time-step. As the simulation progresses, the stripped gas falls further behind the galaxy and the tail becomes more symmetric. In the final time-step, after the outer layers of gas have been removed, the remaining object is left with a very steep rotation curve gradient.

In the plane-of-the-sky case, Fig. 11, a similar asymmetry is observed in the shape of the disc in the second time-step, arising from the gas being slowed by the ICM wind, causing it to fall to higher orbits. This asymmetry shortly vanishes and the tail becomes more collimated by the final time-step. The rotation curves show the velocity curve being 'dragged' along the wind direction to the left and the extreme values of the rotation curve decrease over time. As with the galaxy moving mostly along the line of sight, the velocities in the central region are mostly unaffected and the remaining object retains a steep rotation curve.

Fig. 12 shows the simulated face-on infalling galaxy in 3 timesteps starting from initial infall. The ICM wind direction is downward and slightly inclined away from the observer. The velocity curve of the galaxy shows a disturbed but still fairly symmetric rotation in the initial time-step, with a slight deflection at the edges to higher velocities, similar to that observed in JO201 (cf. fig. 15 within Bellhouse et al. 2017). In the second time-step, more of the gas has been pushed to higher velocities and the central rotation curve 

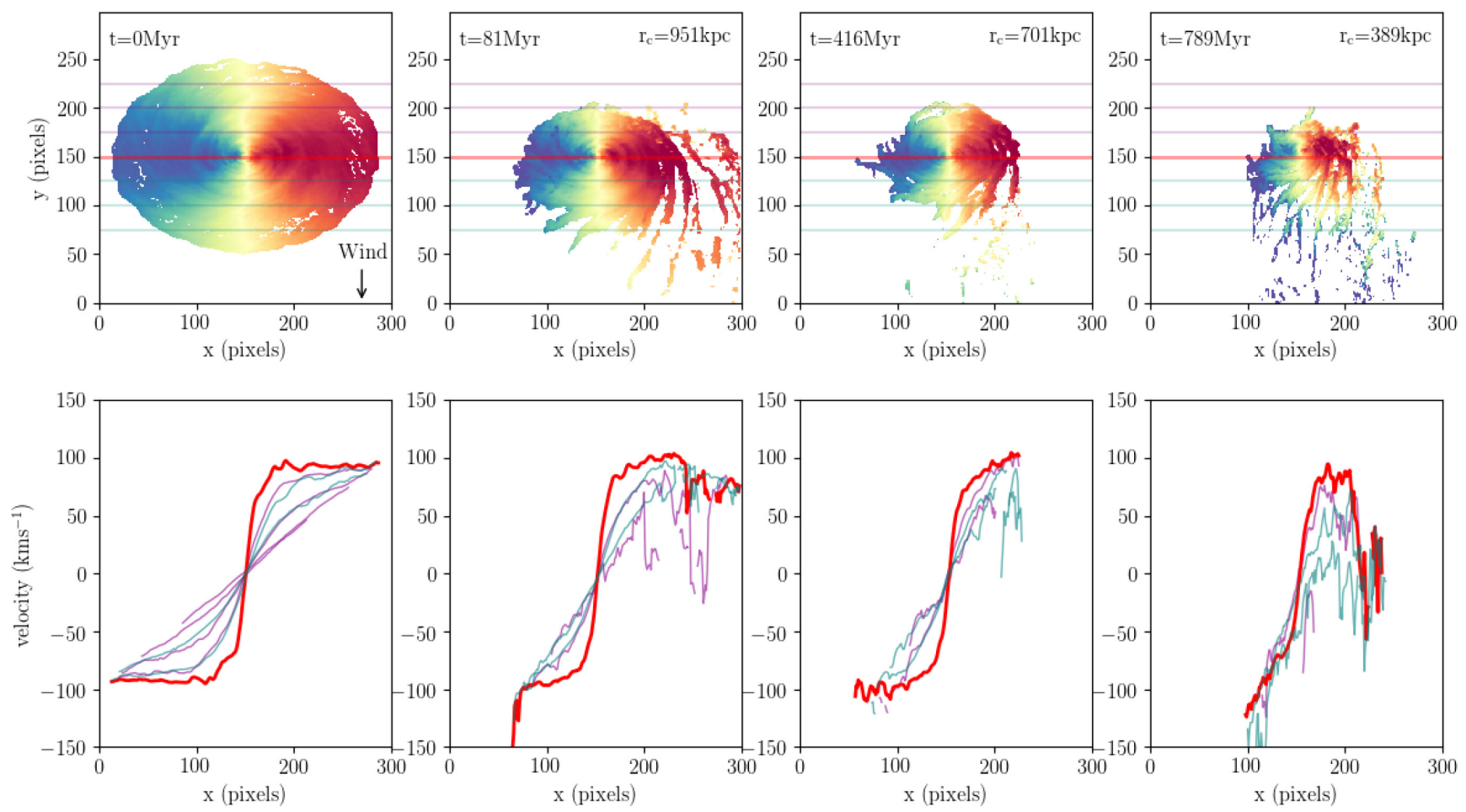

Figure 10. Time-step snapshots of a simulated galaxy undergoing edge-on stripping, inclined $45^{\circ}$ with respect to the observer such that the velocity map can be extracted to emulate a physical observation. In this example, the motion of the galaxy is 50 percent along the line of sight away from the observer, and 50 per cent upward along the plane of the observation. The wind arrow indicates the projected wind direction on the plane of the image; an additional, equal component is towards the observer. The snapshots are labelled with the time since entering the cluster on the upper left, as well as the clustercentric distance in the upper right for each of the infall time-steps. In the lower panels, the red lines show the central rotation curves, while the lighter magenta and cyan lines show the rotation curves across the top and bottom of the disc of the galaxy, respectively, corresponding to the lines on the upper panels.

is noticeably disturbed. By the third time-step, most of the gas has been pushed by stripping to higher velocities and the remaining object retains a steep rotation curve.

These simulations highlight three examples of the effects of orientation of an infalling galaxy with respect to both the ICM wind and the observer, revealing the differences in the effect of face-on and edge-on stripping on the velocity profile. In edge-on stripping scenarios, the differential velocities between the edges of the galaxy rotating into and out of the wind appear to result in a difference in the effectiveness of stripping, such that the gas rotating with the wind direction is more readily stripped, while the gas rotating against the wind is slowed initially, causing an asymmetric build-up of material on the corresponding trailing side.

In the face-on stripping scenario, the gas is stripped around the edges of the disc, causing the rotation curve across all areas of the disc to be much more disturbed; upward deflections on either side of the rotation curve indicate that the stripping occurs evenly around the disc, in contrast with the edge-on scenario. The resulting galaxy after the face-on stripping has a shorter, steeper rotation curve and is fairly compact, likely to be owing to the increased effectiveness of face-on stripping (Abadi, Moore \& Bower 1999; Quilis, Moore \& Bower 2000; Vollmer et al. 2001; Roediger \& Brüggen 2006; Jáchym et al. 2009) removing gas from the disc down to a smaller truncation radius. The differences between the velocity maps in these scenarios suggest that during the phase of unwinding, the morphology of the spiral arm pattern and its underlying kinematics can be a probe of the inclination of a galaxy's stripped gas, and therefore motion, with respect to the ICM.
In all of the simulated galaxies, the final snapshots of Figs 10, 11, and 12 show an extended tail featuring the tentacles and knots commonly observed in stripped galaxies. This suggests that as the residual rotation is slowed and the ICM wind fully washes out the unwinding pattern, the trails of denser gas within the unwound spiral arms become distributed into the tentacles and trails of star-forming knots associated with jellyfish galaxies.

Highlighting the different 'unwinding' processes resulting from edge-on and face-on stripping, 2D flow diagrams were produced to show the motion of the rotating gas. The diagrams were produced by measuring the mean $2 \mathrm{D}$ velocity in bins across the extent of the plane of the disc of each galaxy. The resulting diagrams, shown in Figs 13 and 14, show the mean scalar 2D velocity in the colourmap, along with arrows indicating the $2 \mathrm{D}$ velocity vectors for a subsample of particles in each snapshot. In Fig. 13, the isolated, undisturbed galaxy is shown alongside the edge-on stripped galaxy. The mean 2D velocities in each pixel are calculated for all particles within that pixel bin along the line of sight and the vectors are shown for 500 randomly selected particles in each snapshot. In Fig. 14, showing the face-on stripping galaxies, the flow diagrams are shown for a single snapshot broken into plane-parallel slices along the direction of stripping at intervals of $1 \mathrm{kpc}$. The arrows are drawn for 100 randomly selected particles in each slice. The edge-on stripped galaxy flow diagram further evidences the differential ram pressure caused by the rotation of the galaxy. The gas rotating with the wind, at the bottom edge of the galaxy in the figure, is sped up initially and any gas that remains bound to the galaxy falls to higher orbits conserving angular momentum. The gas rotating into the wind is not completely stripped, 

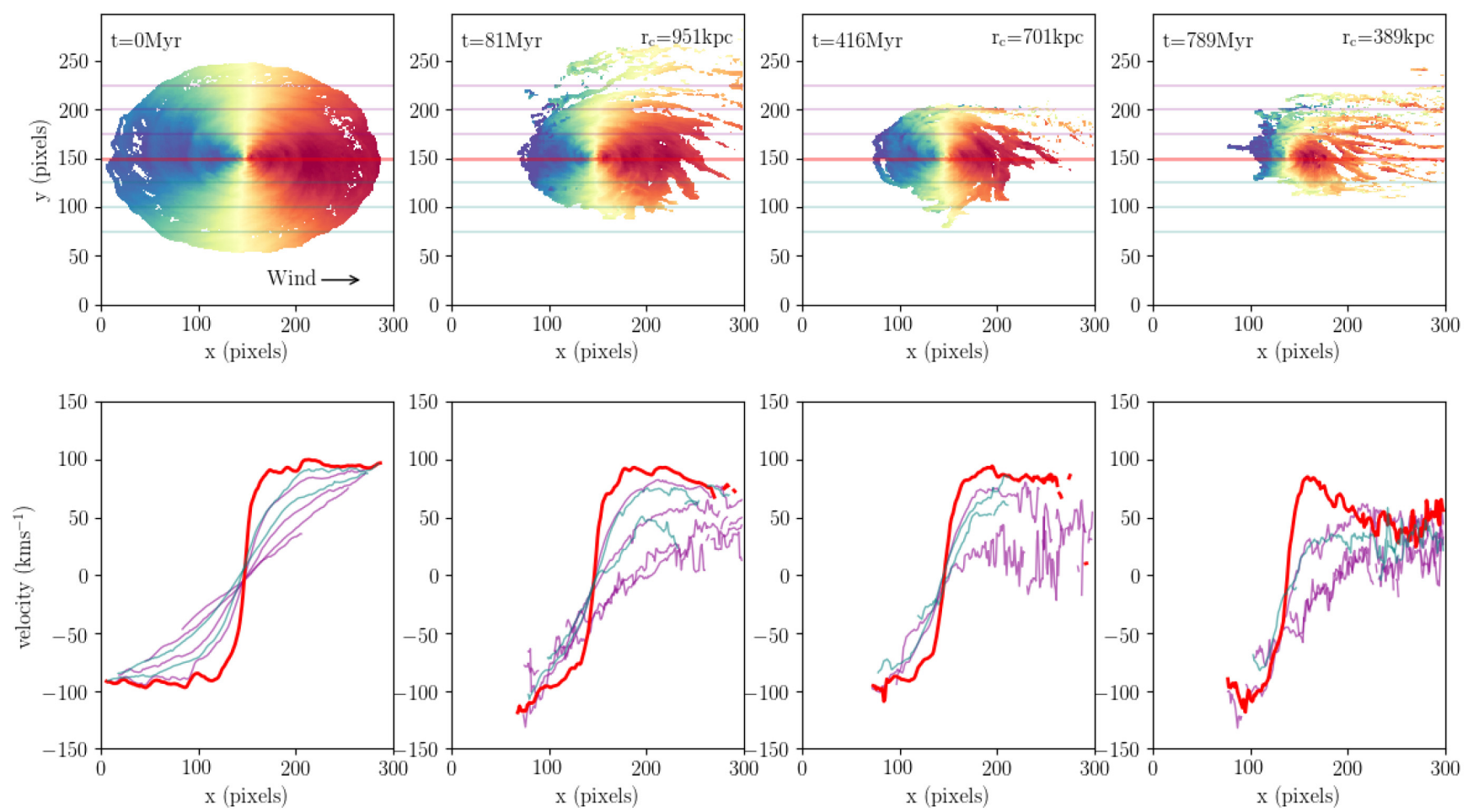

Figure 11. Time-step snapshots of a simulated galaxy undergoing edge-on stripping, inclined $45^{\circ}$ with respect to the observer such that the velocity map can be extracted to emulate a physical observation as for Fig. 10. In this case, the motion of the galaxy is entirely across the plane of the sky, towards the left of the page. The wind arrow indicates the wind direction, which in this case is entirely along the arrow vector, on the plane of the page. The snapshots are labelled with the time since entering the cluster on the upper left, as well as the clustercentric distance in the upper right for each of the infall time-steps. In the lower panels, the red lines show the central rotation curves, while the lighter magenta and cyan lines show the rotation curves across the top and bottom of the disc of the galaxy, respectively, corresponding to the lines on the upper panels.

but slowed by the ram pressure, causing a 'pile-up' of material as the gas continues to rotate. This has the observed effect of the spiral arms opening out and extending on the edge of the galaxy rotating against the wind. Interestingly, the rotational velocity on the leading edge of the disc increases in comparison to the isolated galaxy. This is likely to be due to the gas being pushed deeper into the disc by ram pressure, conserving its angular momentum and increasing its rotational velocity. Furthermore, this figure highlights an important feature of the initial morphology of the tails with respect to the galaxy. In this initial time-step, within the first 81 Myr of stripping, the majority of the 'tail' lies at $45^{\circ}$ to the wind direction due to the ongoing rotation of the stripped material. The majority of observed jellyfish galaxies are seen at more advanced stages of stripping and thus, in projection, the tail directions can be assumed to closely match the wind direction. For galaxies in early stages of infall, however, particularly those with strong edge-on 'unwinding' morphologies, care should be taken when utilizing tail directions to estimate the projected direction of motion.

In Fig. 14, the four panels show slices taken along the direction of stripping, at $1 \mathrm{kpc}$ intervals, for the face-on stripped galaxy. The flow diagram shows the rotation of the gas within $<1 \mathrm{kpc}$ of the galaxy (top left), 1-2 kpc (top right), 2-3 kpc (bottom left), and $>3 \mathrm{kpc}$ (bottom right) from the plane of the disc. The figure clearly shows the expanding ring of stripped material that, once dislodged from the edges of the disc, falls to higher orbits as it becomes further removed from the potential well of the galaxy. This manifests itself in the spiral arm pattern as an extending of the spiral arms, which loosen and unwind as the rotation slows.

\section{SUMMARY AND DISCUSSION}

We find that a pattern of unwinding spiral arms is visible in 11 face-on galaxies in the GASP sample that are undergoing RPS interactions during cluster infall.

The sample of galaxies was selected visually to include galaxies viewed mostly face-on, with clear spiral arms. Out of 12 galaxies that satisfied these first criteria, 11 showed possible signs of unwinding spiral arms. The high incidence of the unwinding effect among faceon viewed galaxies with visible spiral arms in the GASP sample suggests that this effect could be particularly common, although this may be dependent on the original visual sample selection of GASP.

Analysis of the morphology in different wavelengths revealed that this pattern appears only in the stripped $\mathrm{H} \alpha$-emitting ionized gas, and consists of extended, curved trails of material that continue along the lines of the spiral arm patterns. Furthermore, the extent of unwinding around the edges of the disc may indicate the inclination of stripping with respect to the wind, as the more face-on encounters appear to show unwinding around a greater extent of the disc. The morphology and kinematics both reveal that the stripping and unwinding do not affect the older stellar component and the inner discs of the galaxies appear largely undisturbed, confirming that the effect is resulting from purely hydrodynamical processes. Furthermore, with the possible exception of JO69, none of the galaxies in the sample have sufficiently close companions that could plausibly induce a gravitational disturbance.

The radial variations of the pitch angles were compared in each of the stripping and undisturbed galaxies, as well as the simulated stripped galaxies. The sample of stripped galaxies was found to have 

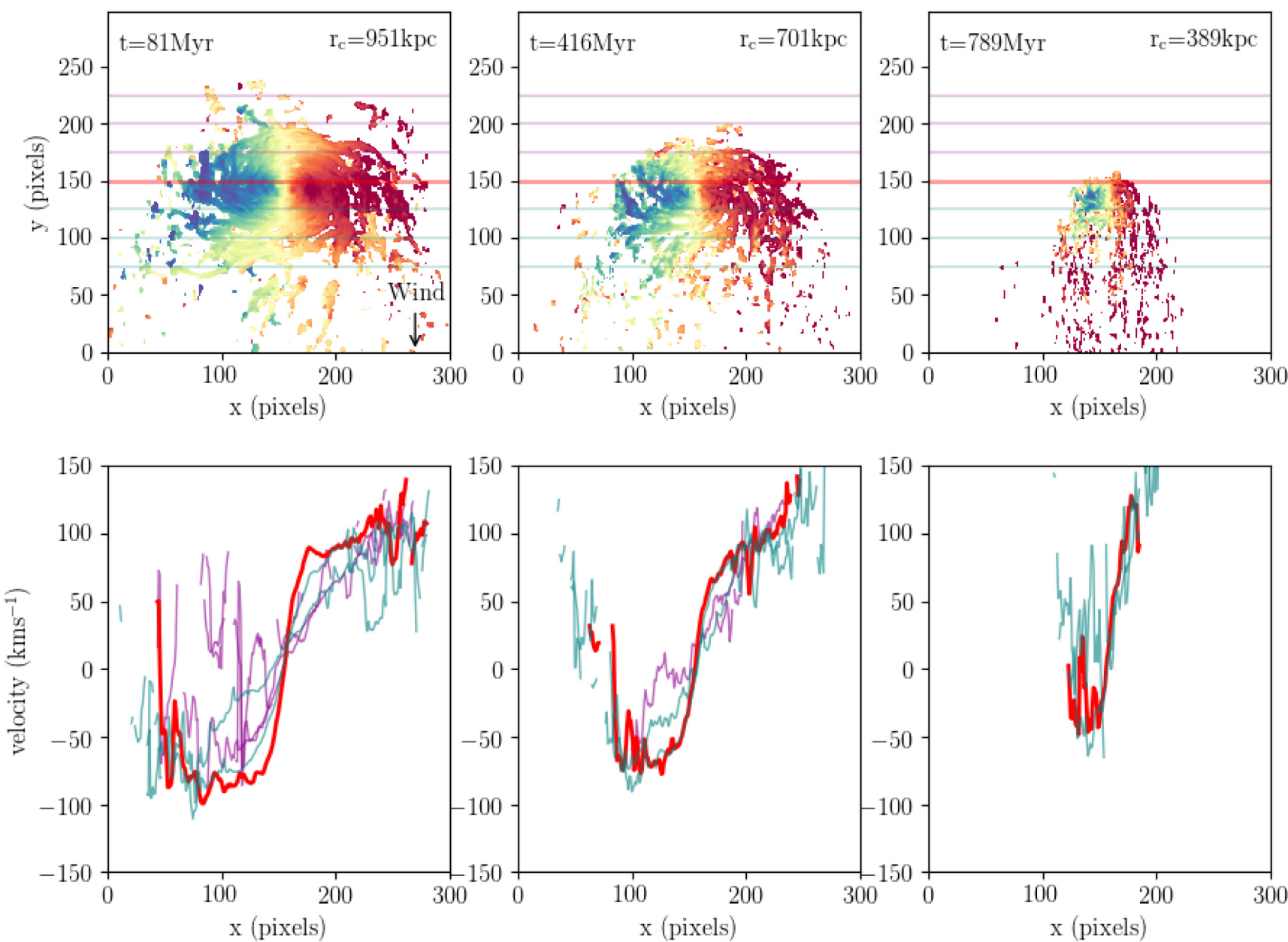

Figure 12. Time-step snapshots of a simulated galaxy undergoing face-on stripping, inclined $45^{\circ}$ with respect to the observer such that the velocity map can be extracted to emulate a physical observation. In this example, the motion of the galaxy is 50 per cent along the line of sight towards the observer, and 50 per cent upward along the plane of the observation. The wind arrow indicates the projected wind direction on the plane of the image; an additional, equal component is away from the observer. The snapshots are labelled with the time since entering the cluster on the upper left, as well as the clustercentric distance in the upper right. In the lower panels, the red lines show the central rotation curves, while the lighter magenta and cyan lines show the rotation curves across the top and bottom of the disc of the galaxy, respectively, corresponding to the lines on the upper panels.

generally higher pitch angles in the outer spiral arms compared to those within two effective radii, in contrast to the six control galaxies that have generally constant pitch angle throughout the disc. This result was also confirmed in the simulated stripped galaxies, which showed similar increases in pitch angle to the observed sample. This confirms that the visual unwinding effect is indeed a pattern in the spiral arm 'opening' in the outermost stripped regions of the galaxy. In a few examples, the pitch angle was found to vary negligibly, which suggests that for those cases, the lengthening of the spiral arms resulting from stripping can also give an appearance of unwinding without actually affecting the pitch angles. Schulz \& Struck (2001) noted possible winding of the inner spiral arms due to compression and 'annealing' of the disc in addition to unwinding of the outer regions of their face-on stripped galaxies. With the given sample, we do not have sufficient evidence to investigate this; however, with a larger sample of galaxies the effect of RPS on the more central spiral arms could be tested.

The unwinding tails were found to be comprised of only the youngest stellar populations, in many cases only stars $<20 \mathrm{Myr}$, when analysed with the SINOPSIS spectrophotometric fitting code. These have likely formed in situ, confirming that the unwinding effect only occurs in the gas component of the galaxy. Moreover, the age gradients observed in some unwinding arms possible capture the process in action, revealing a trail of progressively younger stars left by the opening arms of stripped material.

Simulations were carried out of RPS interactions in different inclinations with respect to the ICM wind to investigate how the unwinding effect can arise and to compare the resulting morphology with observed features in the MUSE sample. The resulting snapshots were analysed throughout the stripping process, and used to produce 'observations' from different viewing angles to compare with the observed sample of galaxies.

The two main effects that can, individually and in combination, give rise to an unwinding pattern are as follows:

(i) In edge-on wind interaction cases, the differential ram pressure in the leading and trailing edges of the disc causes a build-up of material in the leading edge. The tail becomes asymmetric and the slowed rotation of the gas causes the pitch angle to increase.

(ii) In face-on wind interactions, the gas from the outer spiral arms is stripped and withdrawn from the potential well of the galaxy disc, 


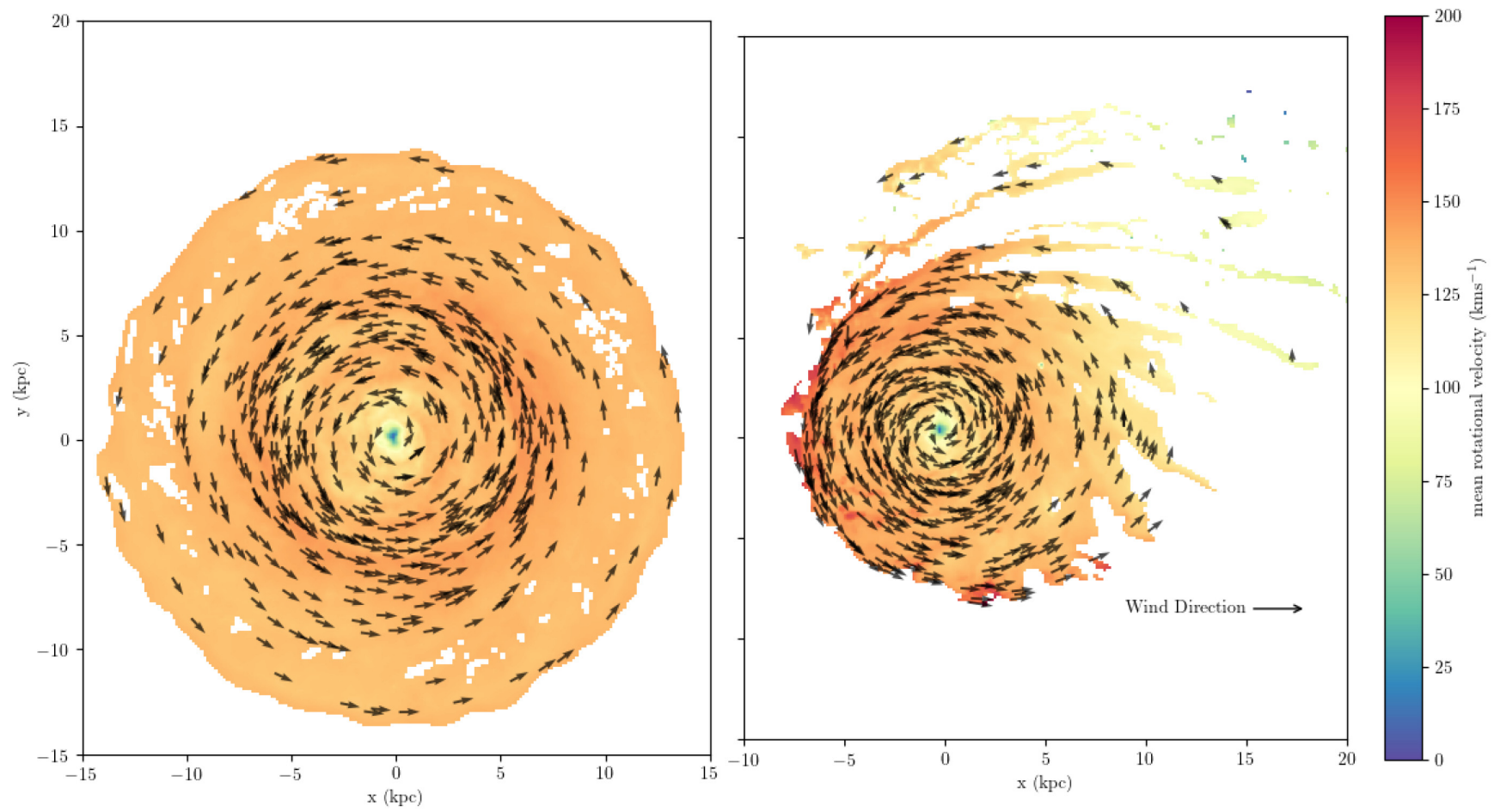

Figure 13. Flow diagram showing the $2 \mathrm{D}$ velocities of gas particles in the isolated (left) and edge-on stripping (right) galaxies. Colours show the mean $2 \mathrm{D}$ velocity in each pixel bin, while arrows indicate the $2 \mathrm{D}$ velocity vector of 500 randomly selected particles.

causing it to move to higher orbits and increase the pitch angle in all directions from the disc.

In most cases, a combination of these two effects will occur as a galaxy is stripped at angles between face-on and edge-on with respect to the ICM wind. Cases in which the galaxy is moving mostly edgeon to the wind appear to be more asymmetric and have tails that are further collimated to one side of the disc, while cases closer to face-on such as JO201 and JO85 show gas extending in a much wider range of directions from the disc.

Furthermore, the simulations, combined with the phase-space positions of our galaxies and the SINOPSIS stellar population analysis, as well as similar effects found in the literature (Steinhauser et al. 2016; Roediger et al. 2014), all point towards the idea that the unwinding effect occurs only for galaxies undergoing first infall on radial plunging orbits, and appears to be a short-lived phase during stripping, containing stars formed in situ no more than $20 \mathrm{Myr}$ ago in our observations and appearing to be fully developed by $81 \mathrm{Myr}$ after first infall in the simulations. After this phase, the simulations suggest that the unwound arm features subsequently evolve over the next $\sim 700$ Myr into the tentacles and trailing knots that are ubiquitous in many other jellyfish galaxies.

It is important to stress that, while we show here that a purely hydrodynamical process can give rise to a pattern of unwinding in the spiral morphology of a galaxy, this does not imply that the unwinding effect is a 'smoking gun' of RPS. It is certainly the case that in an observed unwinding galaxy, effects such as tidal interactions and minor mergers may also be responsible. What we have found here is that simulated galaxies experiencing pure hydrodynamical interactions, in the absence of gravitational effects, can successfully reproduce the morphological and kinematic patterns found in an observed sample of galaxies. It is also important to highlight that the unwinding pattern is not always the sole mark of the interaction and is often compounded with other effects such as compression of the disc and the formation of extended tails and filaments in the wake of the galaxy's motion. The simulations presented here indicate that the unwinding pattern is mostly present in the early stages of stripping, while other effects develop thereafter. The unwinding pattern may therefore be dominant in galaxies initially undergoing stripping and will become washed out as other indicators of stripping gain prevalence, as the galaxy proceeds through infall.

The results of the simulations and the observed differences between patterns in the tails in different regions of phase space suggest that a relation may exist between the stripped morphology and the inclination of the galaxy with respect to the ICM wind, such that broader tails may be indicative of stripping at angles close to faceon to the wind and, vice versa, narrow tails may result from stripping closer to edge-on, as outlined by the two simulated examples in Fig. 9. With a sufficiently large sample, it may be useful to further explore the possibility of such a relation.

An important key result of this study is that we confirm through simulations and observations that hydrodynamical interactions alone can produce an unwinding effect in spiral galaxies, consisting of curved tails and arcs of stripped material resulting from the residual rotation of the stripped material; RPS can produce not only straight tentacles, but also arced tentacles under the right conditions. Since curved tails and stripped arcs can be indicative of tidal interactions, care should be taken to confirm the origin of stripping when classifying samples of interacting galaxies based on these particular morphological features. This is particularly important when considering selection of ram-pressure stripped galaxies in broad-band imaging data by manual visual inspection or automated feature detection; galaxies that might otherwise be dismissed due to 'tidal-like' features should be carefully checked. In our sample, we noted that all of the galaxies exhibited some other signatures of stripping; however, the simulations revealed that certain orientations 

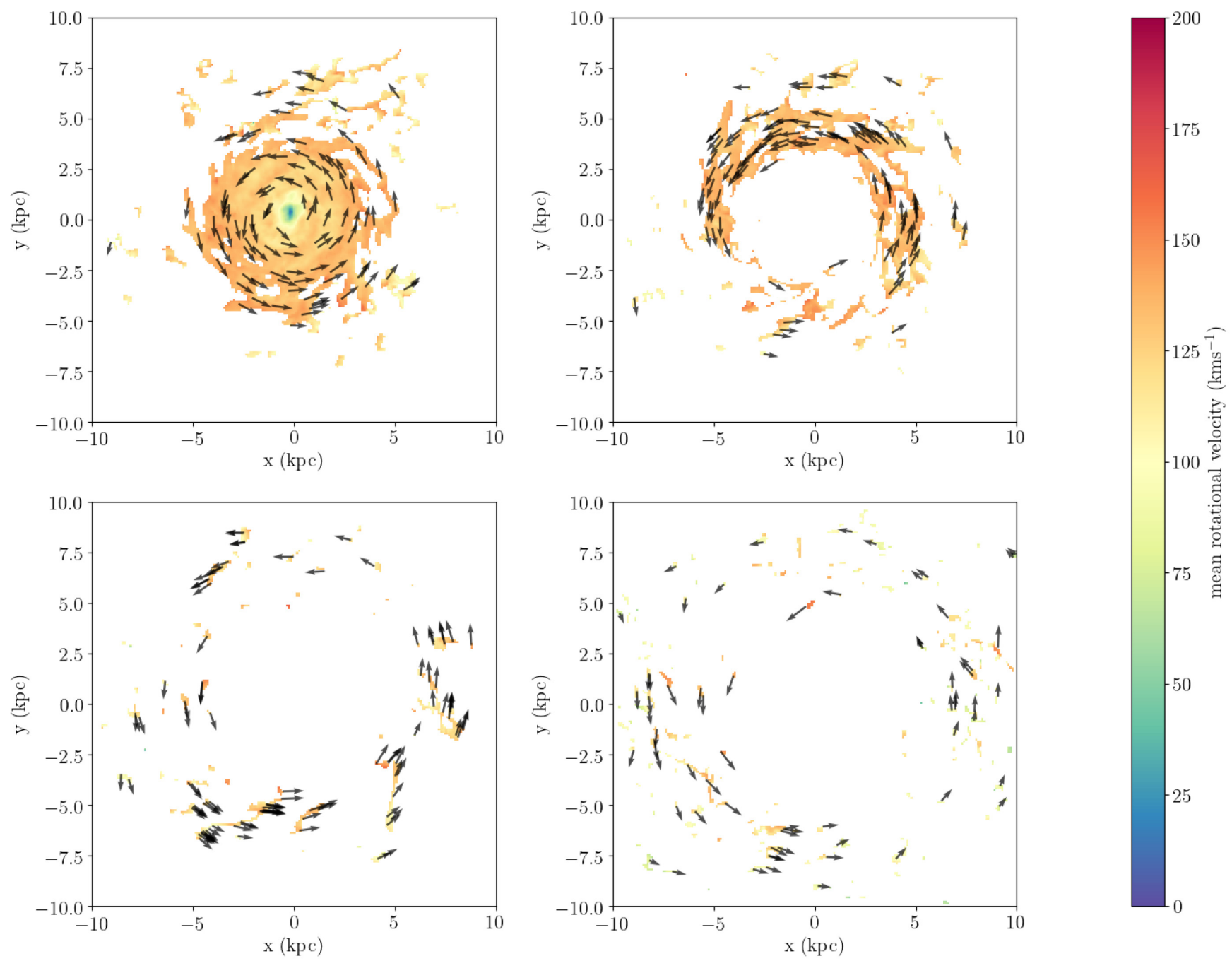

Figure 14. Flow diagram showing the $2 \mathrm{D}$ velocities of gas particles in four slices of the galaxy undergoing face-on stripping, taken at different distances from the plane of the galaxy. Top left: $<1 \mathrm{kpc}$, top right: $1-2 \mathrm{kpc}$, bottom left: $2-3 \mathrm{kpc}$, and bottom right: $>3 \mathrm{kpc}$. Colours show the mean $2 \mathrm{D}$ velocity in each pixel bin, while arrows indicate the $2 \mathrm{D}$ velocity vector of 100 randomly selected particles in each slice.

can give rise to unwinding with no other obvious effects, which could plausibly be interpreted as evidence of a gravitational, not hydrodynamical, interaction. In cases where no obvious companion is present to confirm gravitational interactions, observations of the stellar and gas rotation curves are likely the best indicators of the true cause of morphological disturbance.

\section{ACKNOWLEDGEMENTS}

Based on observations collected at the European Organization for Astronomical Research in the Southern hemisphere under ESO programme 196.B-0578. This project has received funding from the European Research Council (ERC) under the European Union's Horizon 2020 research and innovation programme (grant agreement no. 833824).

CB would like to thank Frederic Vogt for valuable discussion and guidance in developing the azimuthal reprojection plots, based on figures shown in Vogt et al. (2017).

YJ acknowledges financial support from CONICYT PAI (Concurso Nacional de Inserción en la Academia 2017) No. 79170132 and FONDECYT (Fondo Nacional de Desarrollo Científico y Tecnológico) Iniciación 2018 No. 11180558.

We acknowledge financial contribution from the contract ASI-INAF n.2017-14-H.0, from the grant PRIN MIUR 2017 n.20173ML3WW_001 (PI Cimatti) and from the INAF (Istituto Nazionale di Astrofisica) main-stream funding programme (PI Vulcani).

\section{DATA AVAILABILITY}

The data underlying this article are available at http://archive.eso.or g/scienceportal/home under programme ID 196.B-0578.

\section{REFERENCES}

Abadi M. G., Moore B., Bower R. G., 1999, MNRAS, 308, 947

Bacon R. et al., 2010, in Ian S. M., Suzanne K. R., Hideki T., eds, Proc. SPIE Conf. Ser. Vol. 7735, Ground-Based and Airborne Instrumentation for Astronomy III. SPIE, Bellingham, p. 773508

Behroozi P. S., Wechsler R. H., Wu H.-Y., 2013, ApJ, 762, 109

Bellhouse C. et al., 2017, ApJ, 844, 49

Bellhouse C. et al., 2019, MNRAS, 485, 1157 
Boselli A., Gavazzi G., 2006, PASP, 118, 517

Boselli A. et al., 2018, A\&A, 615, A114

Byrd G., Valtonen M., 1990, ApJ, 350, 89

Calvi R., Poggianti B. M., Vulcani B., 2011, MNRAS, 416, 727

Cappellari M., Emsellem E., 2004, PASP, 116, 138

Cardelli J. A., Clayton G. C., Mathis J. S., 1989, ApJ, 345, 245

Chabrier G., 2003, PASP, 115, 763

Cramer W. J., Kenney J. D. P., Sun M., Crowl H., Yagi M., Jáchym P., Roediger E., Waldron W., 2019, ApJ, 870, 63

Fasano G. et al., 2006, A\&A, 445, 805

Fossati M. et al., 2018, A\&A, 614, A57

Franchetto A. et al., 2020, ApJ, 895, 106

Fritz J. et al., 2017, ApJ, 848, 132

Fumagalli M., Fossati M., Hau G. K. T., Gavazzi G., Bower R., Sun M., Boselli A., 2014, MNRAS, 445, 4335

George K. et al., 2018, MNRAS, 479, 4126

George K. et al., 2019, MNRAS, 487, 3102

Gullieuszik M. et al., 2015, A\&A, 581, A41

Gunn J. E., Gott III J. R., 1972, ApJ, 176, 1

Hahn O., Abel T., 2011, MNRAS, 415, 2101

Ho I.-T. et al., 2014, MNRAS, 444, 3894

Jáchym P., Köppen J., Palouš J., Combes F., 2009, A\&A, 500, 693

Jáchym P. et al., 2019, ApJ, 883, 145

Jaffé Y. L. et al., 2018, MNRAS, 476, 4753

Kenney J. D. P., Koopmann R. A., 1999, AJ, 117, 181

Kenney J. D. P., Geha M., Jáchym P., Crowl H. H., Dague W., Chung A., van Gorkom J., Vollmer B., 2014, ApJ, 780, 119

Lewis A., Challinor A., Lasenby A., 2000, ApJ, 538, 473

Merritt D., 1983, ApJ, 264, 24

Mihos J. C., Bothun G. D., Richstone D. O., 1993, ApJ, 418, 82

Moretti A. et al., 2014, A\&A, 564, A138

Moretti A. et al., 2017, A\&A, 599, A81

Moretti A. et al., 2018, MNRAS, 475, 4055

Perret V., Renaud F., Epinat B., Amram P., Bournaud F., Contini T., Teyssier R., Lambert J. C., 2014, A\&A, 562, A1

Poggianti B. M. et al., 2016, AJ, 151, 78

Poggianti B. M. et al., 2017a, Nature, 548, 304

Poggianti B. M. et al., 2017b, ApJ, 844, 48

Poggianti B. M. et al., 2019a, MNRAS, 482, 4466

Poggianti B. M. et al., 2019b, ApJ, 887, 155

Quilis V., Moore B., Bower R., 2000, Science, 288, 1617

Radovich M., Poggianti B., Jaffé Y. L., Moretti A., Bettoni D., Gullieuszik M., Vulcani B., Fritz J., 2019, MNRAS, 486, 486
Ramatsoku M. et al., 2020, A\&A, 640, A22

Rasmussen J., Ponman T. J., Mulchaey J. S., 2006, MNRAS, 370, 453

Roediger E., Brüggen M., 2006, MNRAS, 369, 567

Roediger E., Brüggen M., Owers M. S., Ebeling H., Sun M., 2014, MNRAS, 443, L114

Schulz S., Struck C., 2001, MNRAS, 328, 185

Sheen Y.-K. et al., 2017, ApJ, 840, L7

Spitzer L., Jr, Baade W., 1951, ApJ, 113, 413

Springel V., 2000, MNRAS, 312, 859

Springel V. et al., 2005, Nature, 435, 629

Steinhauser D., Schindler S., Springel V., 2016, A\&A, 591, A51

Teyssier R., 2002, A\&A, 385, 337

Tinsley B. M., Larson R. B., 1979, MNRAS, 186, 503

Tomičić N. et al., 2018, ApJ, 869, L38

Toomre A., 1977, in Tinsley B. M., Larson D., Campbell R. B. G., eds, Evolution of Galaxies and Stellar Populations. Yale Univ. Obs., New Haven, CT, USA, p. 401

Valluri M., 1993, ApJ, 408, 57

Veilleux S., Cecil G., Bland-Hawthorn J., 2005, ARA\&A, 43, 769

Vogt F. P. A., Pérez E., Dopita M. A., Verdes-Montenegro L., Borthakur S., 2017, A\&A, 601, A61

Vollmer B., Cayatte V., Balkowski C., Duschl W. J., 2001, ApJ, 561, 708

Vulcani B. et al., 2018, ApJ, 866, L25

Vulcani B. et al., 2019, MNRAS, 487, 2278

Vulcani B. et al., 2020, ApJ, 892, 146

Wolter A., Esposito P., Mapelli M., Pizzolato F., Ripamonti E., 2015, MNRAS, 448, 781

Yoshida M. et al., 2008, ApJ, 688, 918

\section{APPENDIX A: KINEMATICS COMPARISONS OF GALAXY SAMPLE}

This appendix follows on from Fig. 2 with the velocity map comparisons for the rest of the sample of unwinding galaxies, with the left-hand panels showing the $\mathrm{H} \alpha$-emitting ionized gas velocity and the right-hand panels showing the stellar velocities, revealing that the majority of the disturbance is visible only in the stripped gas and not in the old stellar component. This is indicative that the disturbance is hydrodynamical in nature and not resulting from gravitational effects. 

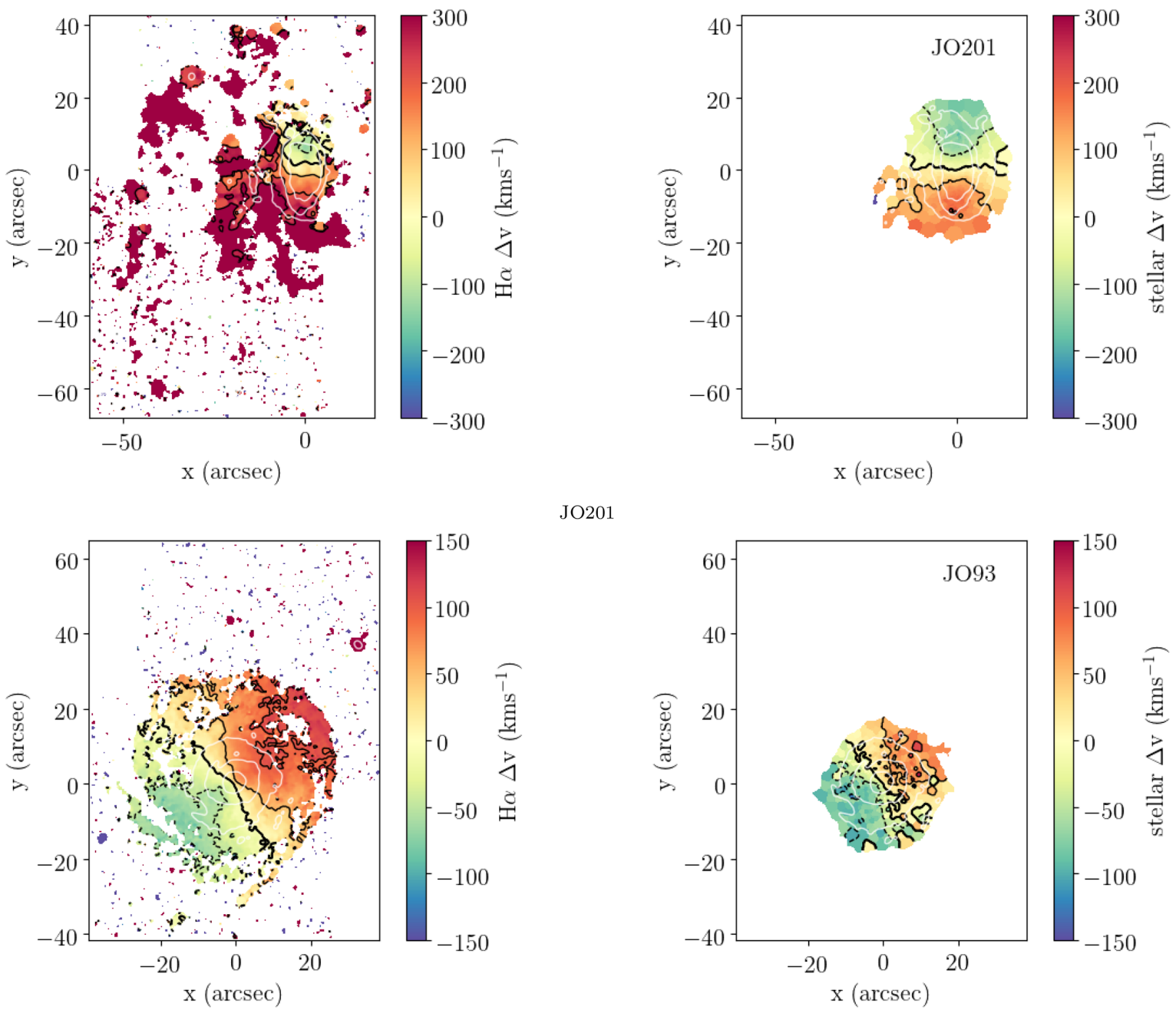

JO201
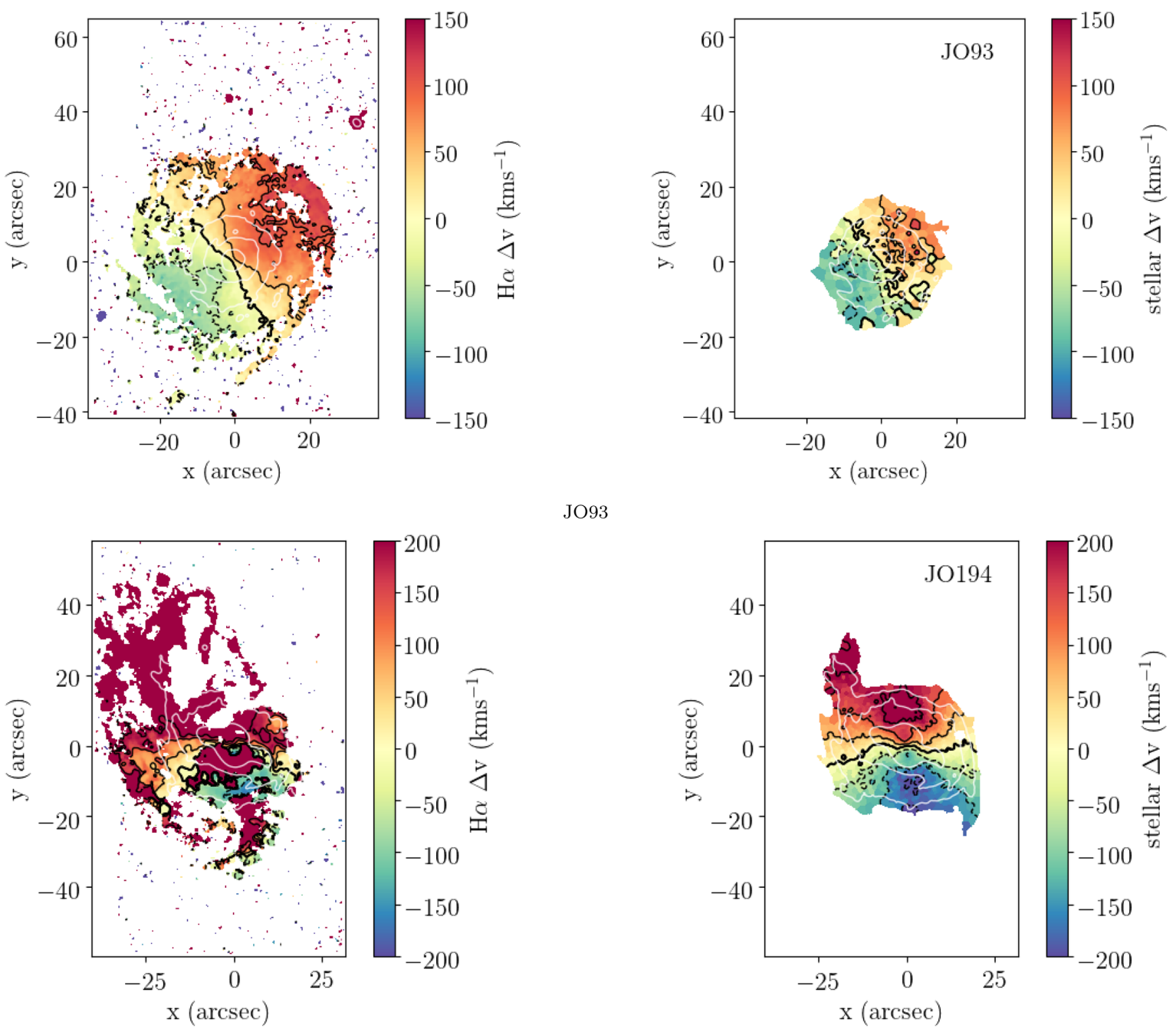

JO93

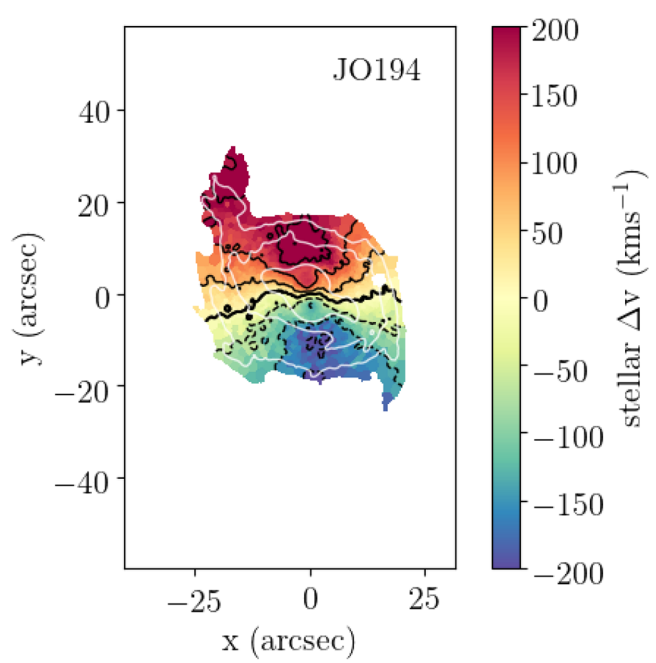

JO194

Figure A1. Comparison of kinematics maps in the rest of the sample, following from Fig. 2. Black lines indicate iso-velocity contours on the corresponding maps. The left-hand panels show the ionized gas velocity maps for each galaxy measured from emission-line kinematics. The right-hand panels show the stellar velocity maps measured using PPXF. White contours denote stellar continuum isophotes. 
Unwinding spirals in GASP

1305
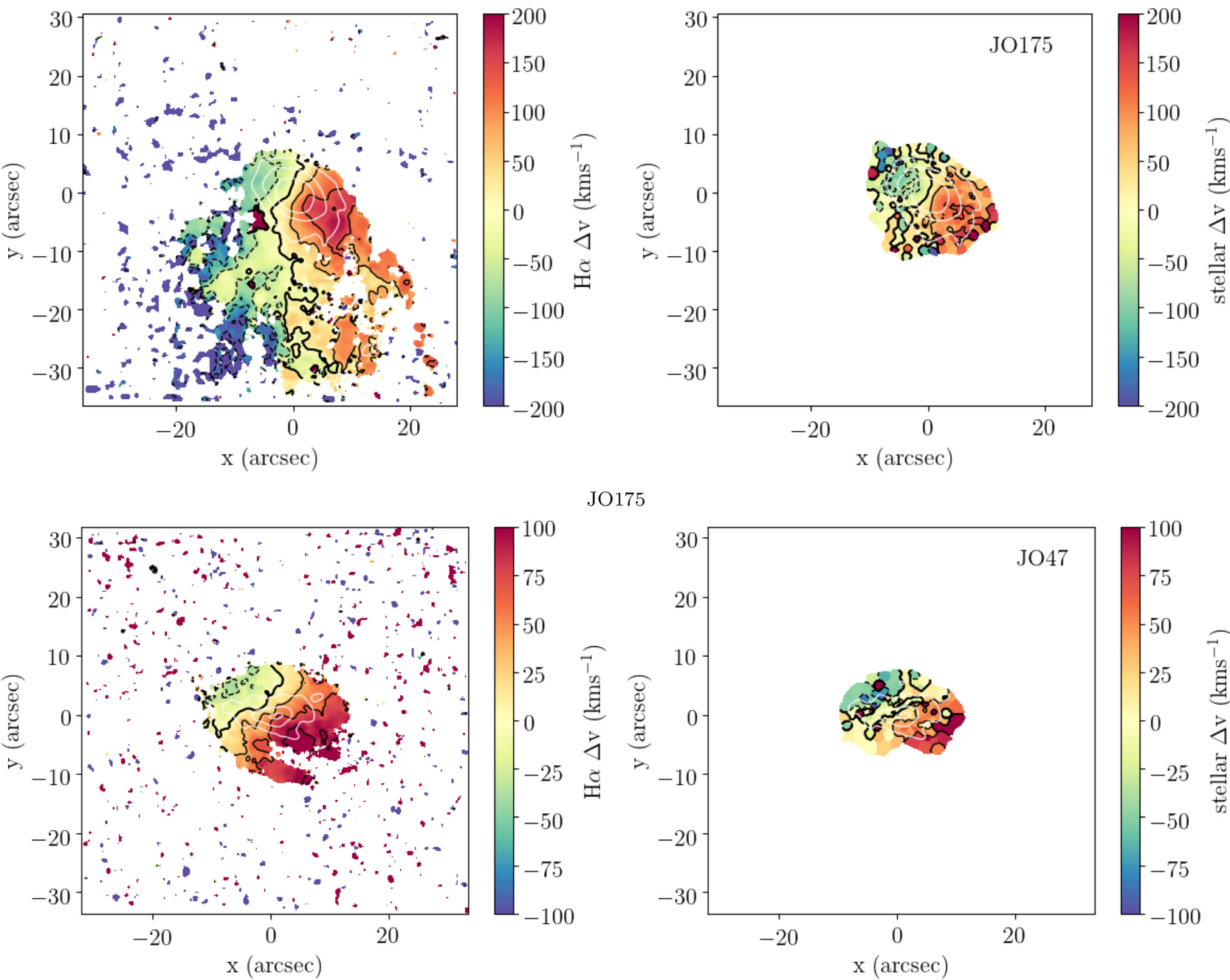

JO175
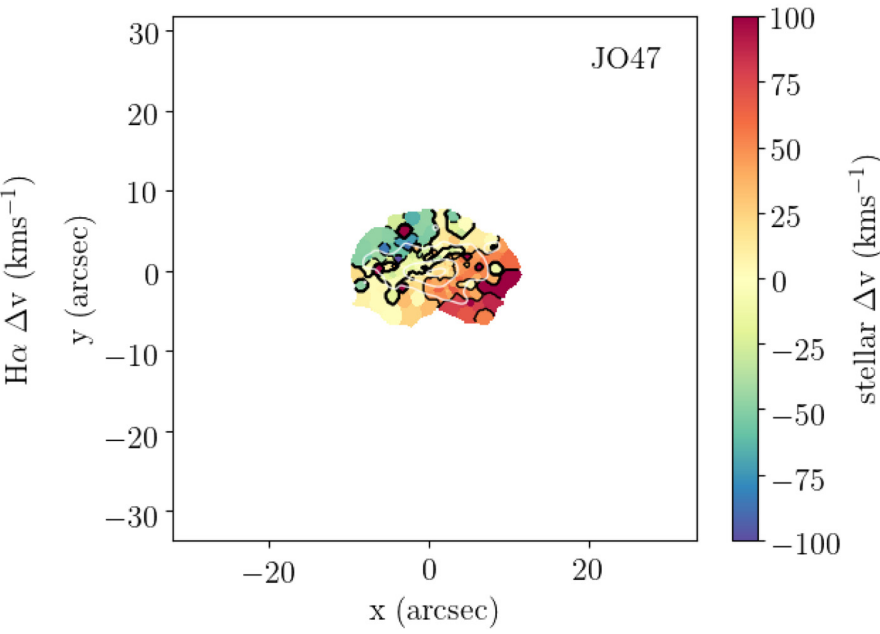

JO47
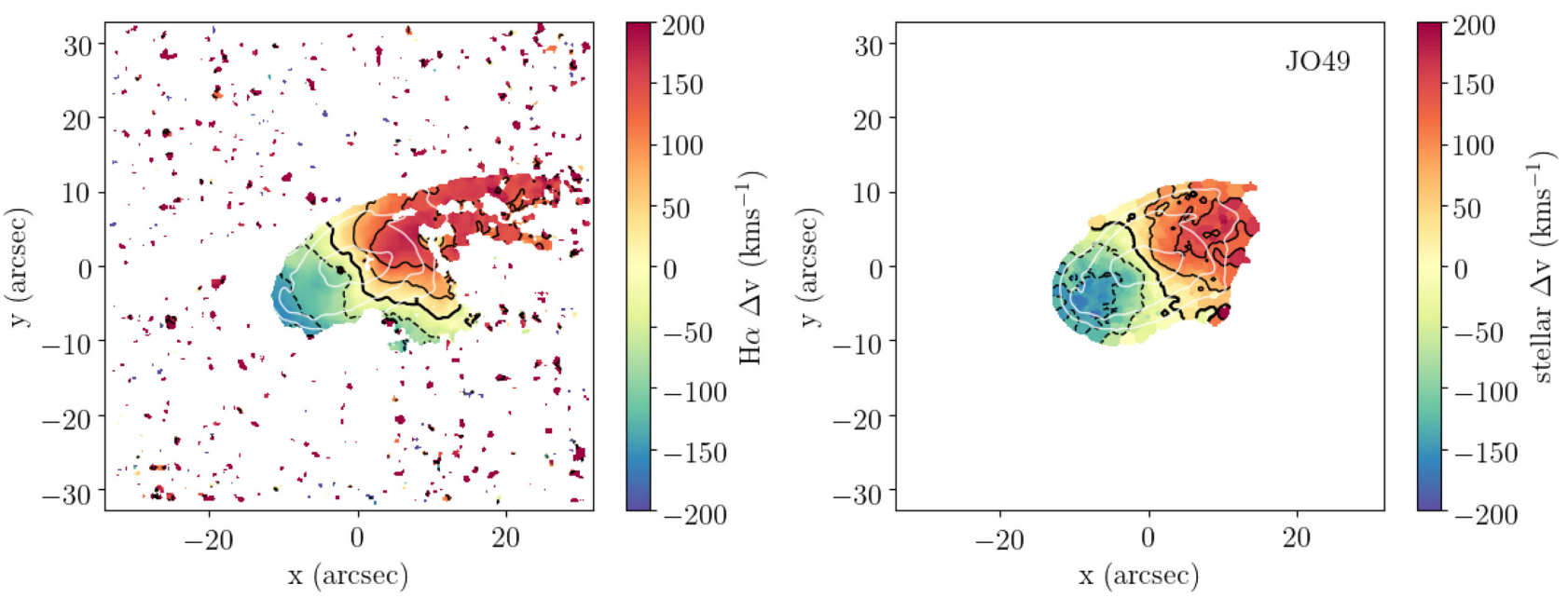

JO49

Figure A1 - continued. 

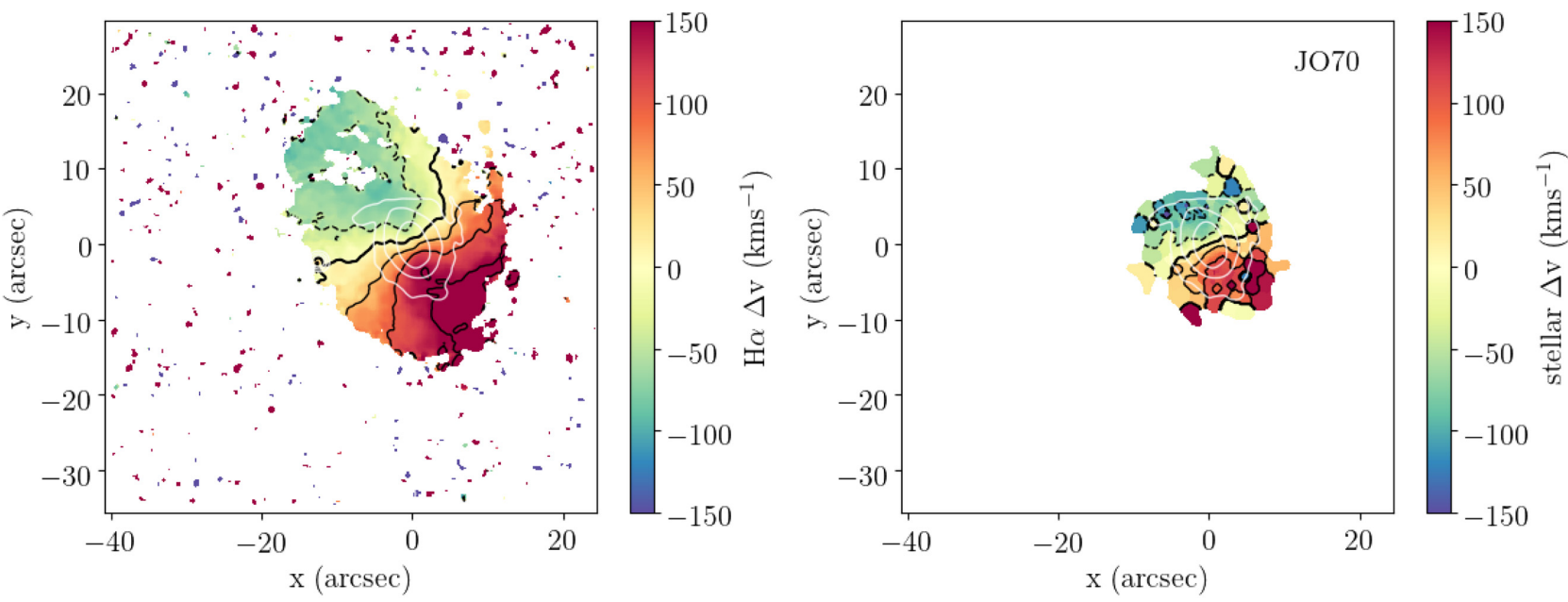

JO70
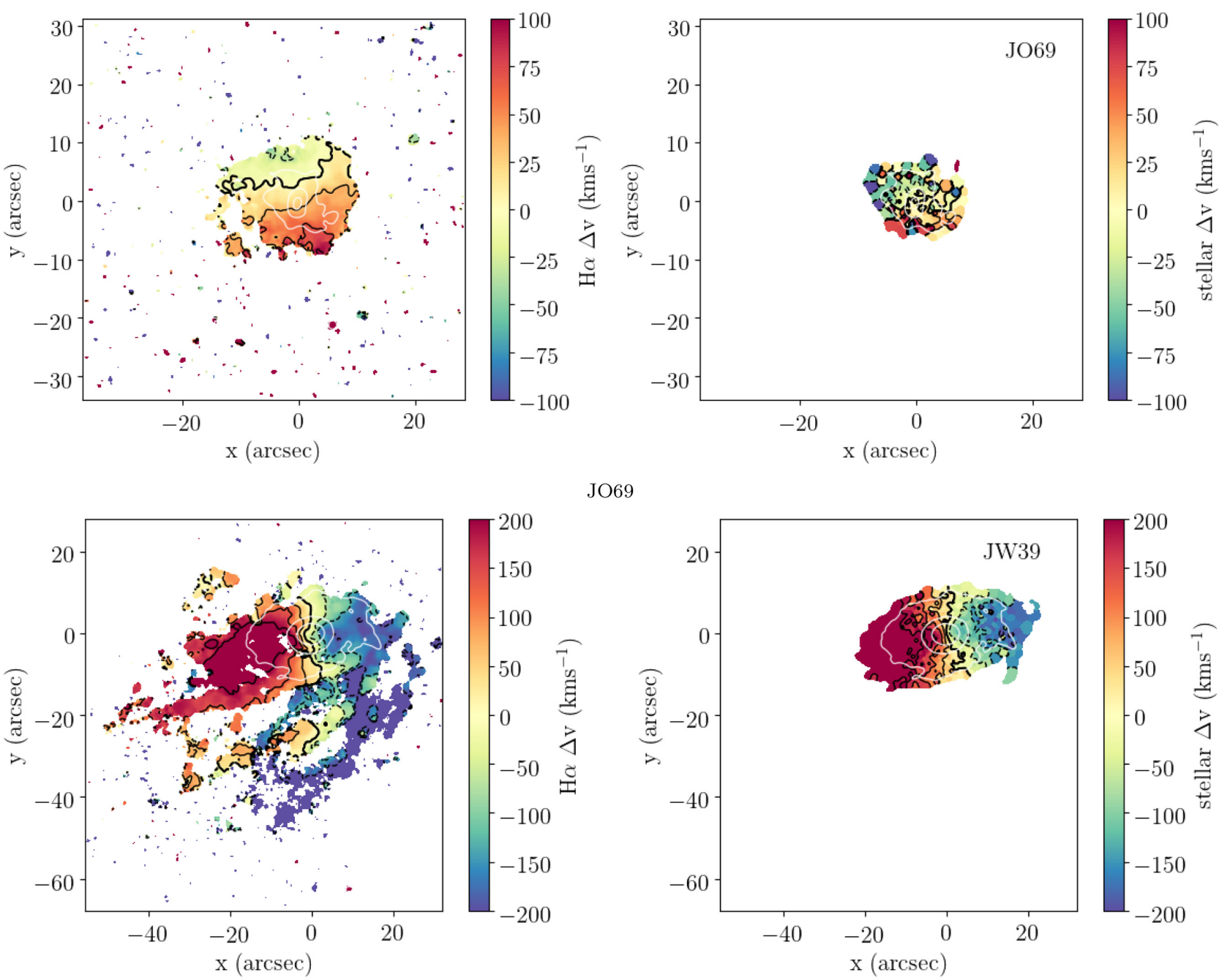

JO69

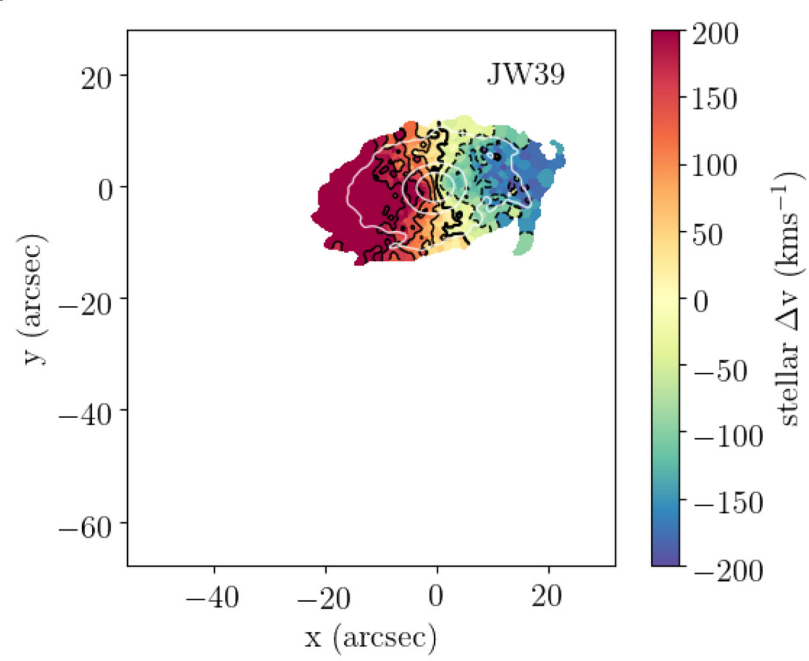

JW39

Figure A1 - continued 


\section{APPENDIX B: AZIMUTHAL DIAGRAMS FOR SIMULATED GALAXIES}

Similarly to the azimuthal figures of the 'unwrapped' discs of JO85, JO200, and P25500, we produced azimuthal figures of the 'observed' simulated galaxies at the first time-step (81 Myr after entering the cluster). The galaxies were rotated to be viewed face-on, before the average density was integrated in bins along the line of sight. These were then treated in the same way as the observed galaxies, reprojecting each pixel into polar space and manually tracing the spiral arms to obtain the pitch angles. The resulting plots are shown in Fig. B1.

\section{APPENDIX C: AZIMUTHAL DIAGRAMS FOR FULL SAMPLE}

We show in this appendix the azimuthal figures as presented in Section 4.1 for the remainder of the sample. The values of the pitch angles measured in the inner and outer discs of each galaxy were averaged and given in Table 2 .
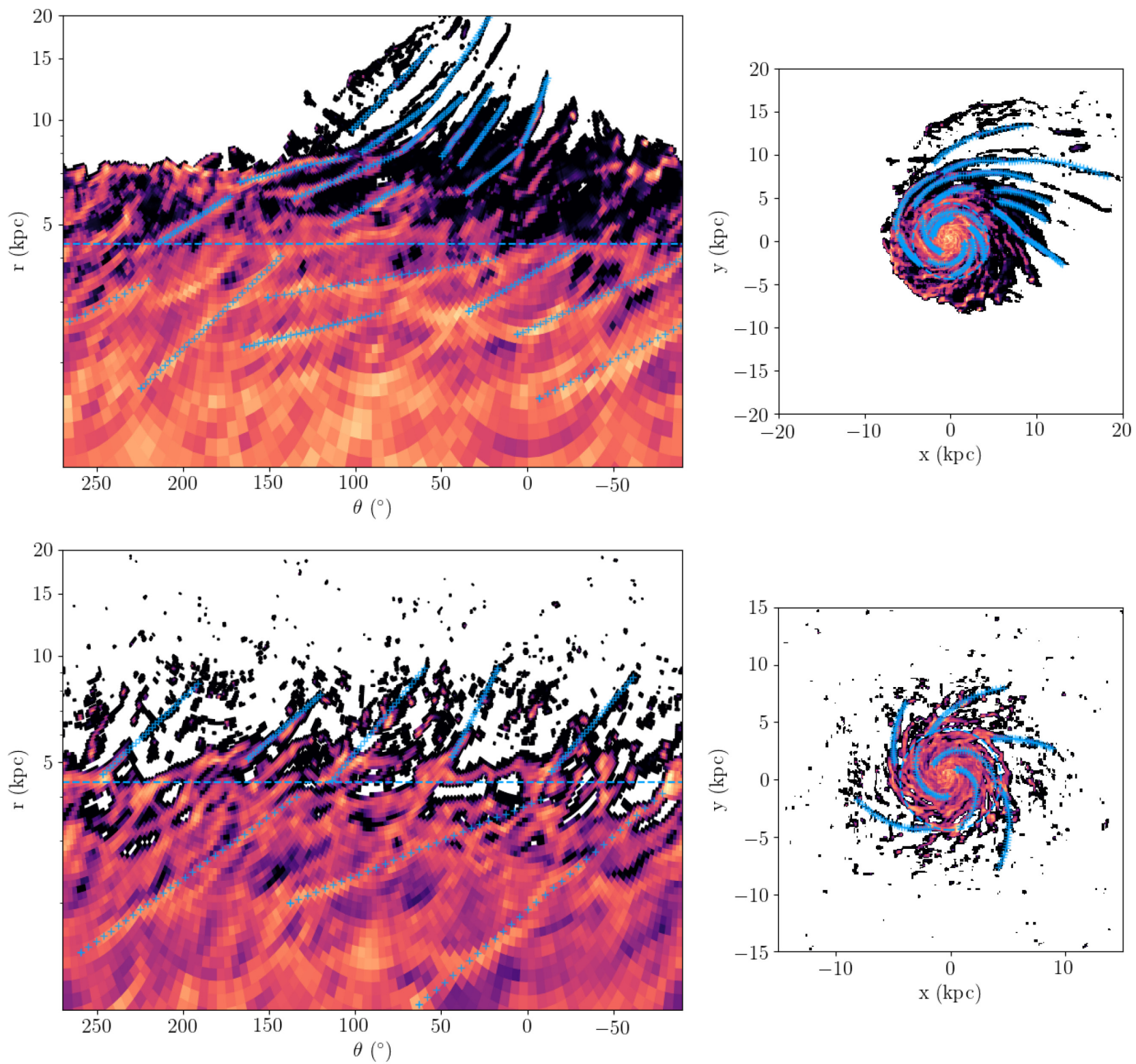

Figure B1. Azimuthal plots of $\mathrm{H} \alpha$ for the 'unwrapped' discs of the edge-on stripping (top) and face-on stripping (bottom) simulated galaxies, showing radial projected distance from the centre in logarithmic scale against azimuthal angle in the left-hand panels, alongside the original galaxy discs on the right-hand panels. The blue dashed horizontal lines on the left-hand panels and the dashed blue ellipses on the right mark 2 scale radii on the disc. Lines of + symbols mark spiral arm patterns identified by eye on the unwrapped figure and are shown reprojected back on the original galaxy discs. The pitch angles of these spiral arms are shown along with their average radial distance in Fig. 6. 
JO201
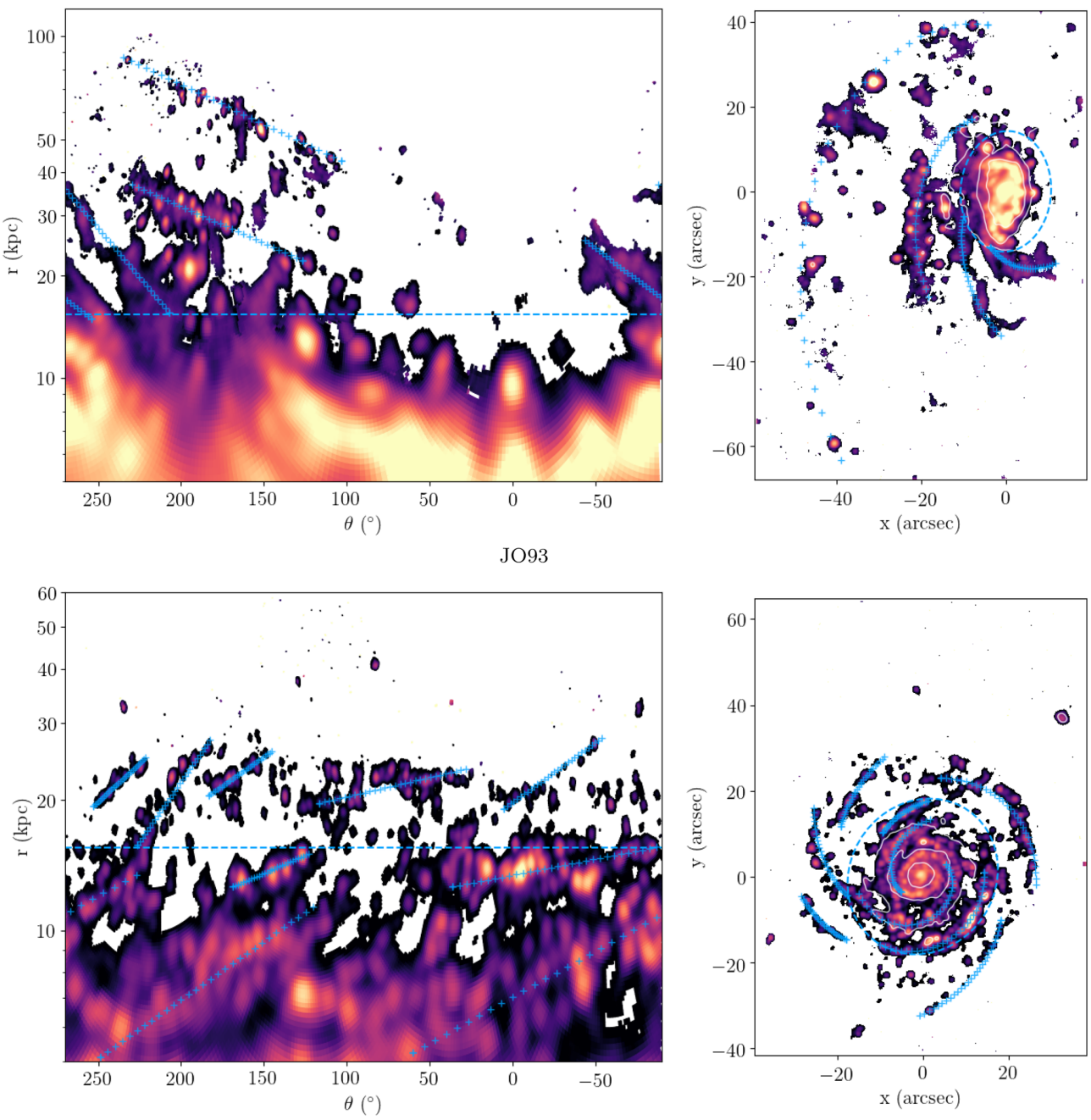

JO194
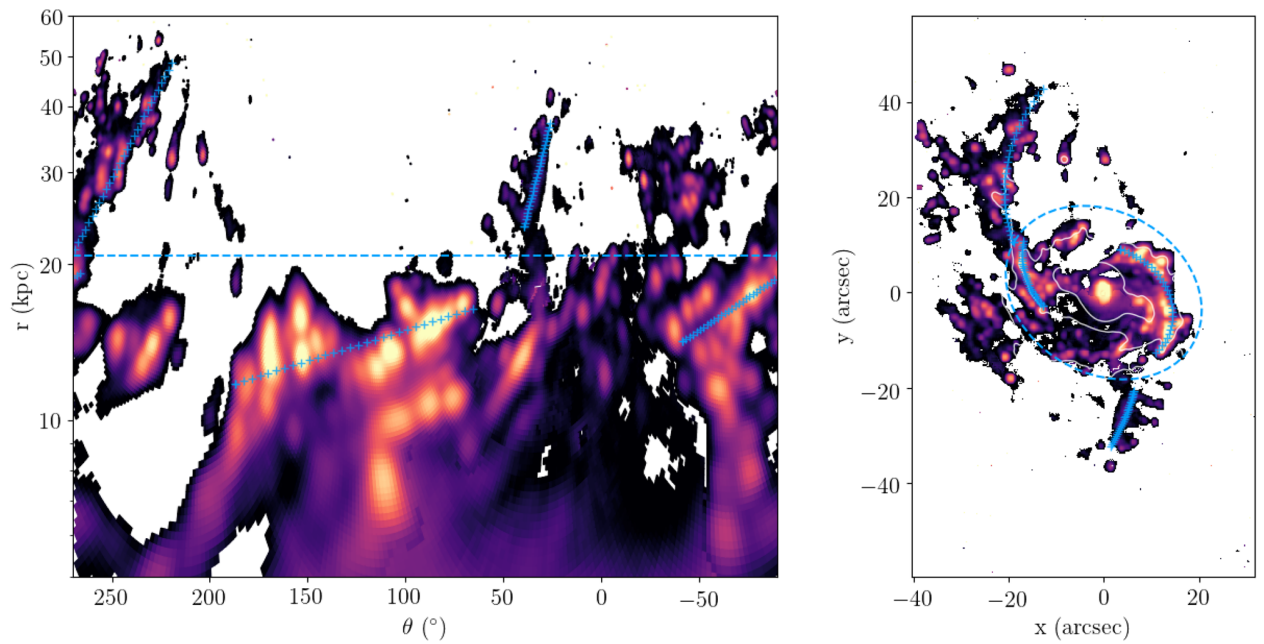

Figure C1. Azimuthal plots of $\mathrm{H} \alpha$ for the 'unwrapped' discs of the remainder of the unwinding sample and control galaxies, showing radial projected distance from the centre in logarithmic scale against azimuthal angle in the left-hand panels, alongside the original galaxy discs on the right-hand panels. The white contours on the right-hand panels denote stellar continuum isophotes. The blue dashed horizontal lines on the left-hand panels and the dashed blue ellipses on the right mark two effective radii on the disc. Lines of + symbols mark spiral arm patterns identified by eye on the unwrapped figure and are shown reprojected back on the original galaxy discs. The pitch angles of these spiral arms are shown along with their average radial distance in Fig. 6. 
JO175
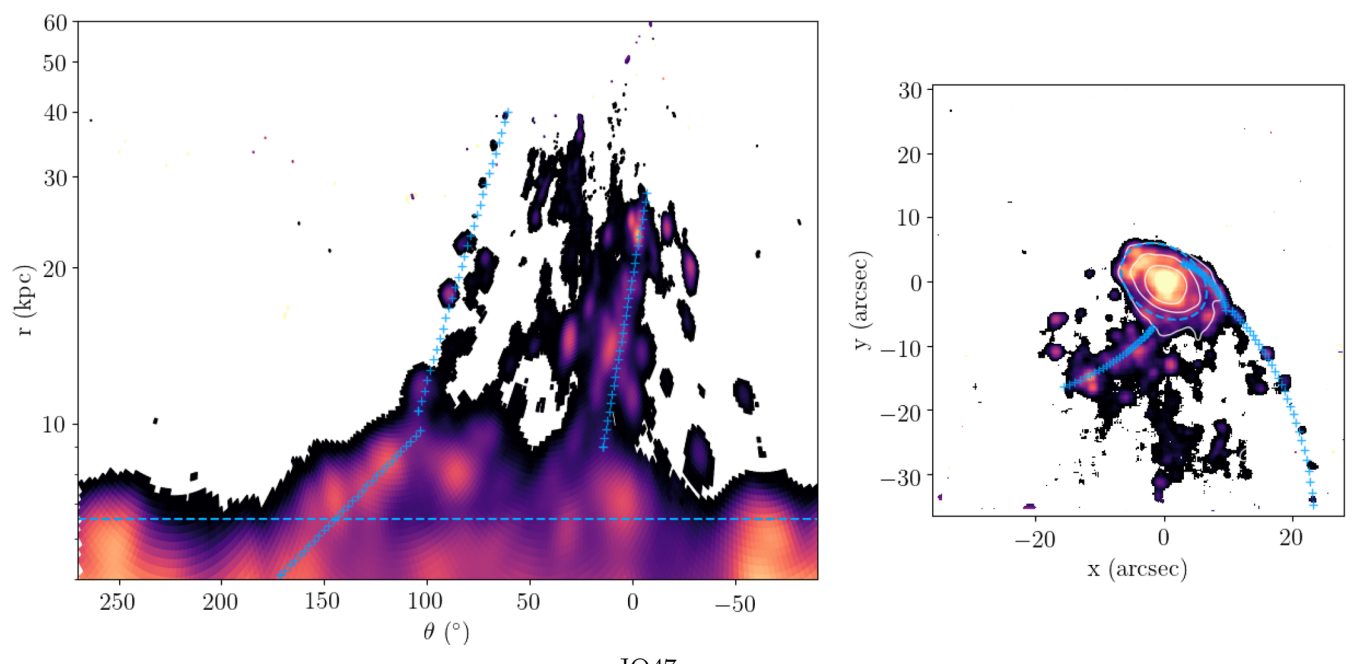

JO47
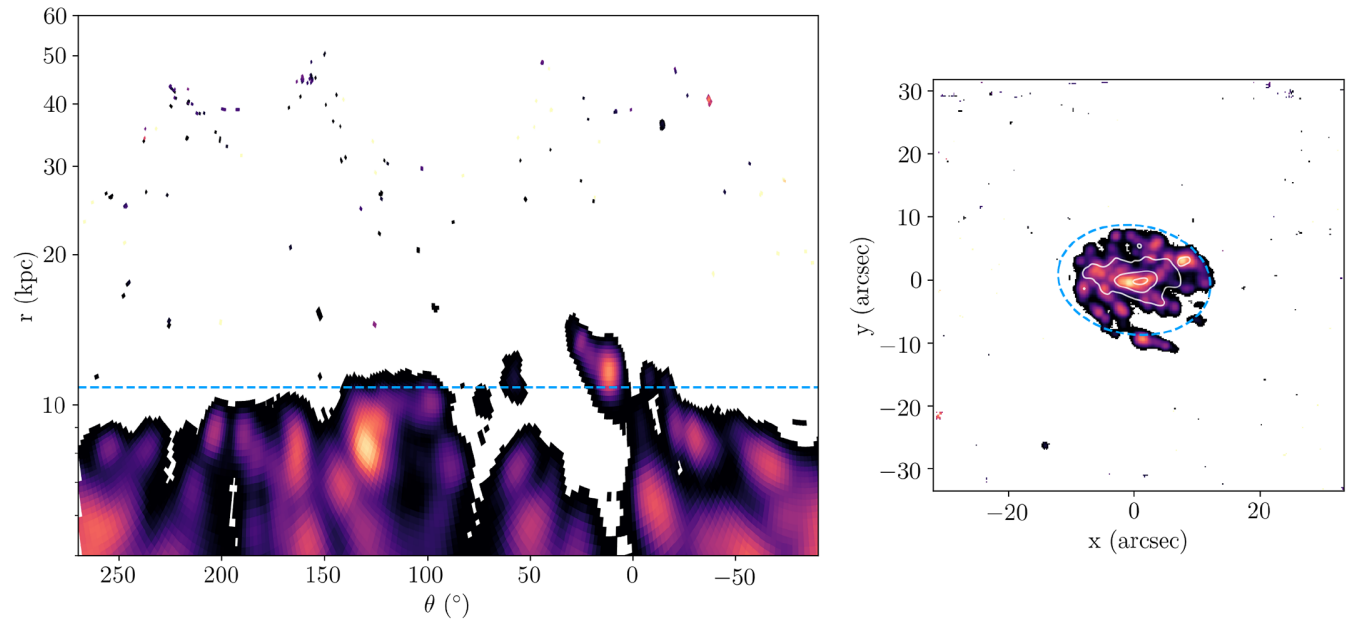

JO49
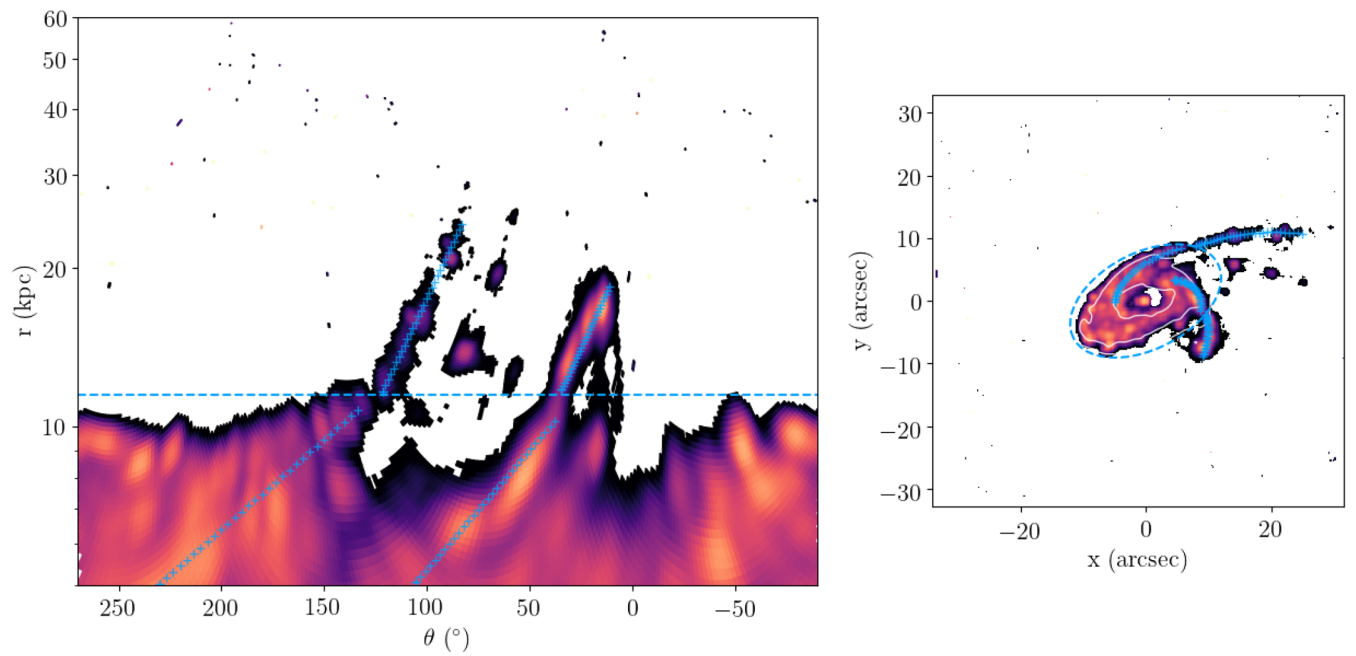

Figure C1 - continued. 

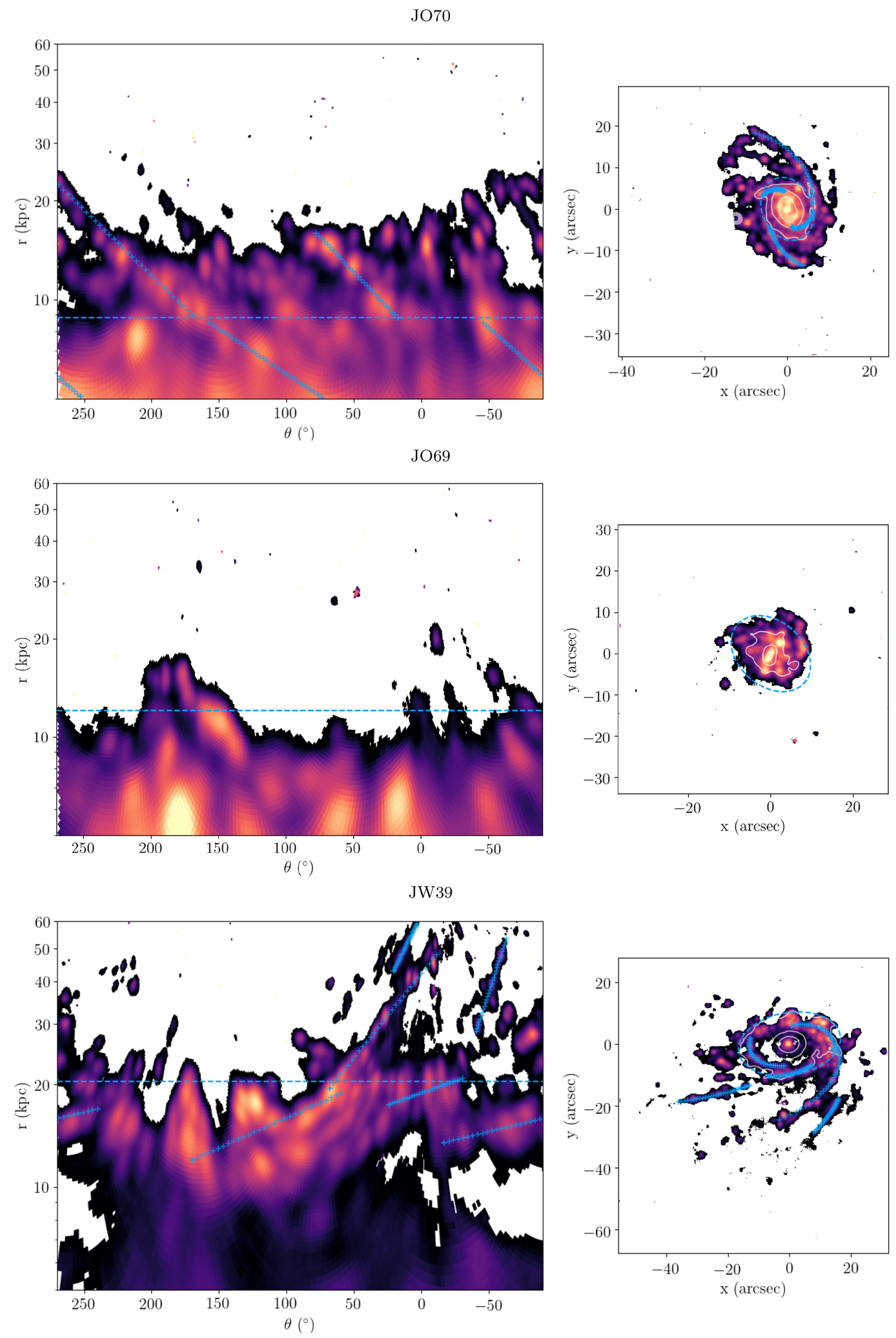

Figure C1 - continued. 
P21734
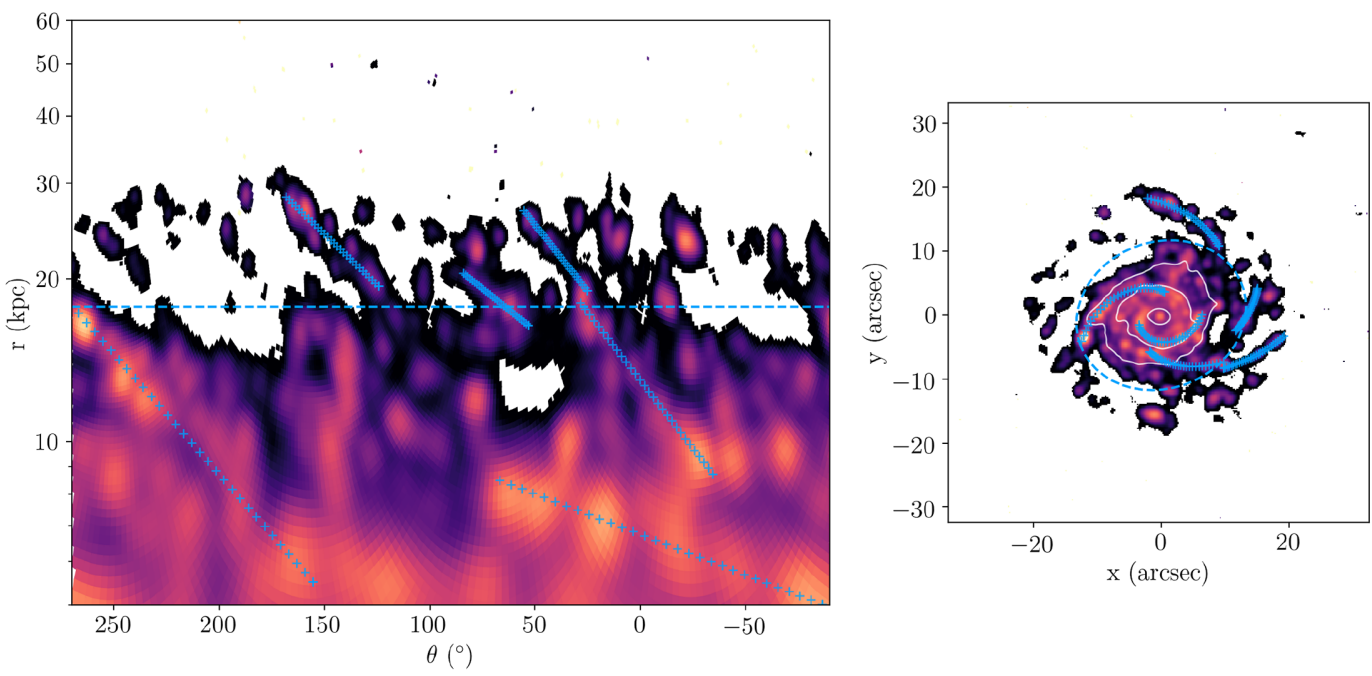

P48157
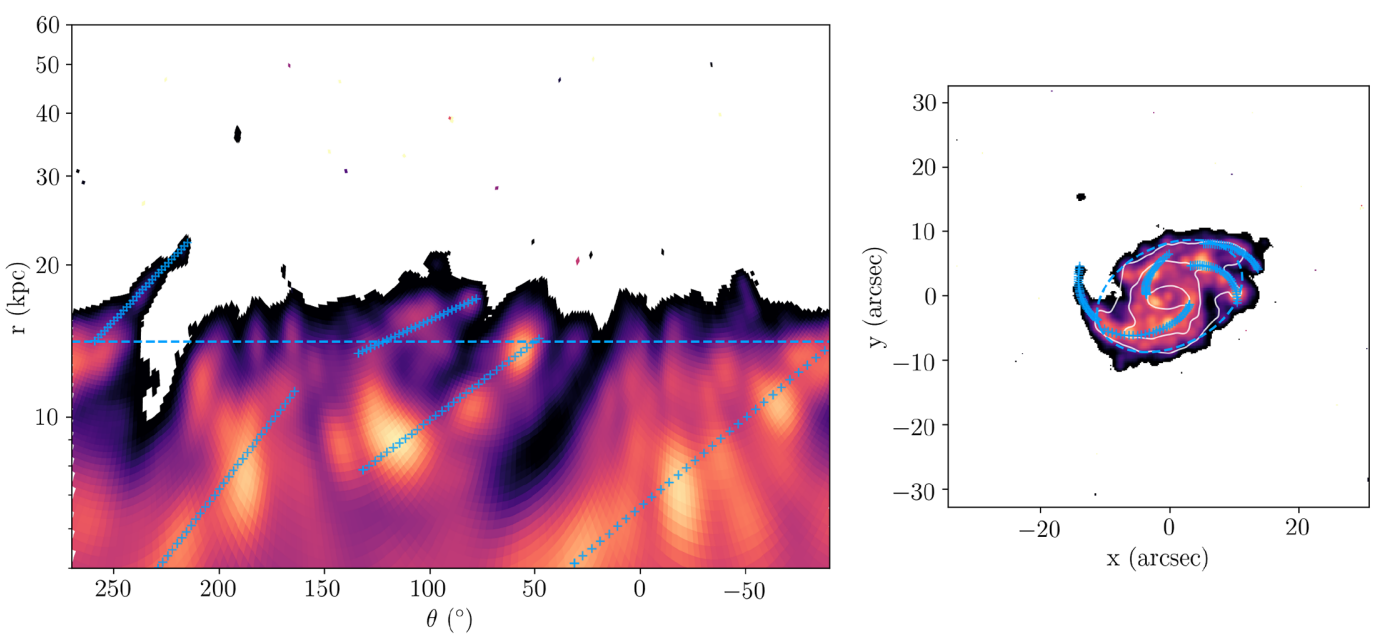

P19482
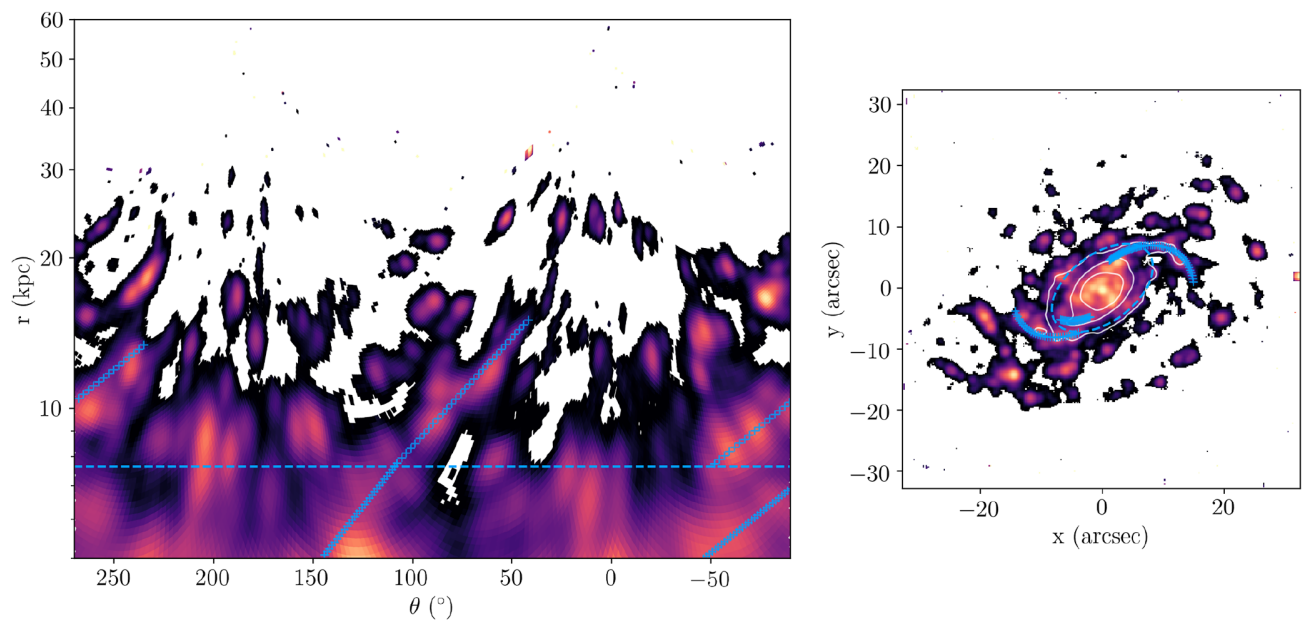

Figure C1 - continued. 
P63661

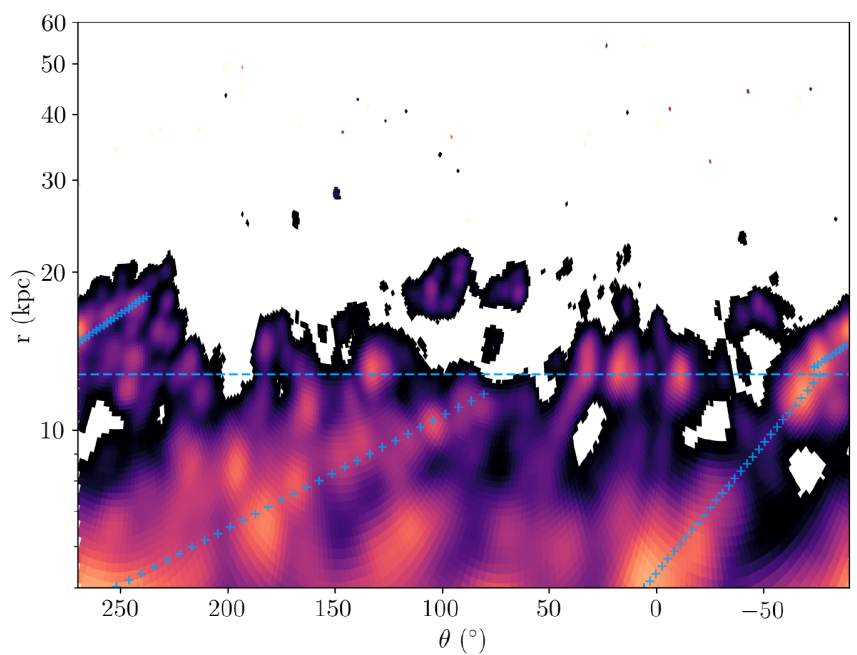

P95080

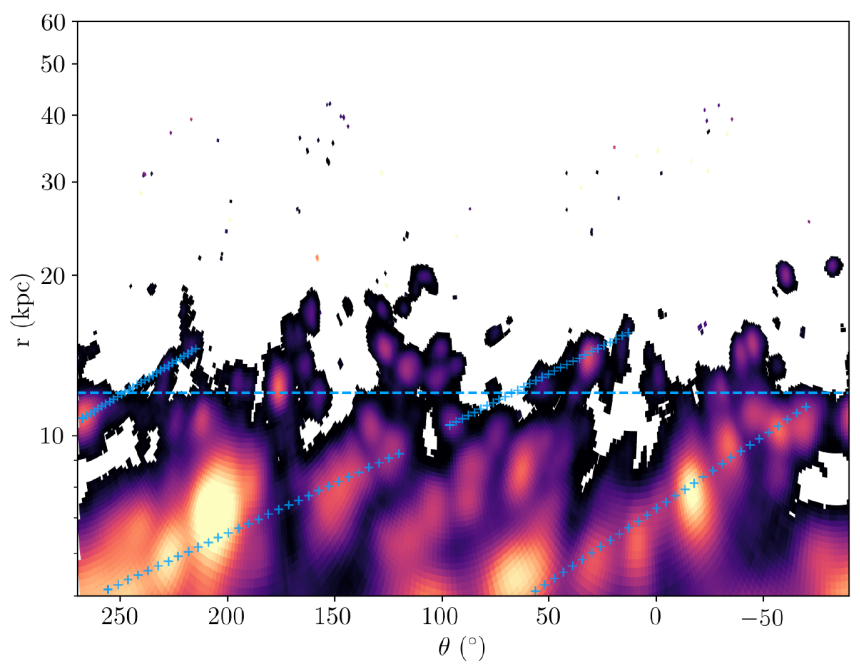

Figure C1 - continued.
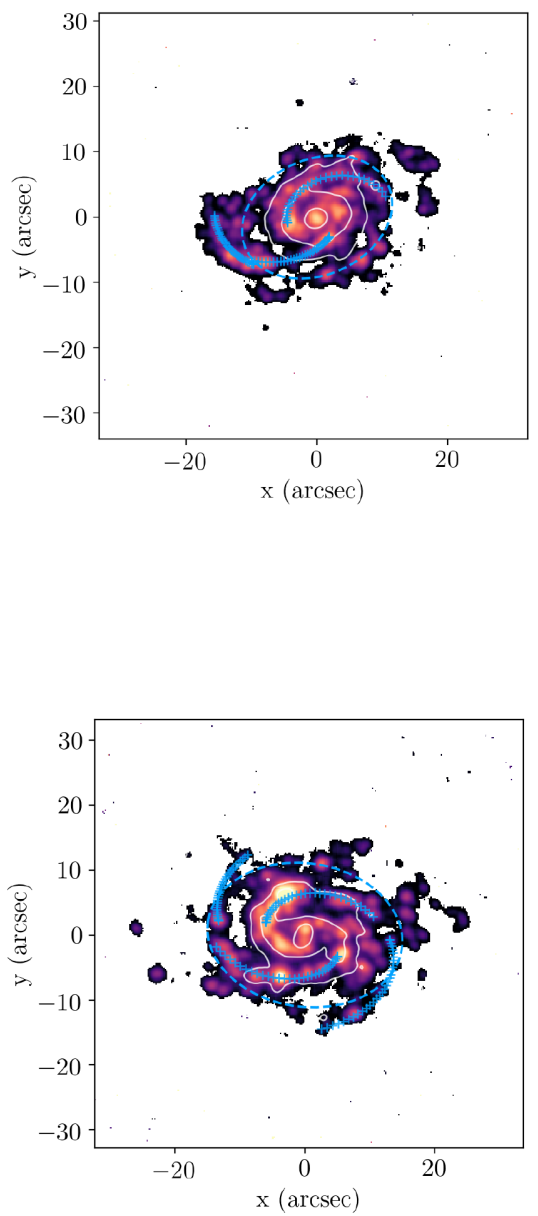

This paper has been typeset from a $\mathrm{T}_{\mathrm{E}} \mathrm{X} / \mathrm{L} \mathrm{T}_{\mathrm{E}} \mathrm{X}$ file prepared by the author. 